\title{
Measurements of the average properties of a suspension of bubbles rising in a vertical channel
}

\author{
By ROBERTO ZENIT ${ }^{1}$, \\ DONALD L. KOCH ${ }^{1}$ AND ASHOK S. SANGANI \\ ${ }^{1}$ School of Chemical Engineering, Cornell University, Ithaca, NY 14853, USA \\ ${ }^{2}$ Department of Chemical Engineering and Materials Science, Syracuse University, \\ Syracuse, NY 13244, USA
}

(Received 31 January 2000 and in revised form 6 September 2000)

Experiments were performed in a vertical channel to study the behaviour of a monodisperse bubble suspension for which the dual limit of large Reynolds number and small Weber number was satisfied. Measurements of the liquid-phase velocity fluctuations were obtained with a hot-wire anemometer. The gas volume fraction, bubble velocity, bubble velocity fluctuations and bubble collision rate were measured using a dual impedance probe. Digital image analysis was performed to quantify the small polydispersity of the bubbles as well as the bubble shape.

A rapid decrease in bubble velocity with bubble concentration in very dilute suspensions is attributed to the effects of bubble-wall collisions. The more gradual subsequent hindering of bubble motion is in qualitative agreement with the predictions of Spelt \& Sangani (1998) for the effects of potential-flow bubble-bubble interactions on the mean velocity. The ratio of the bubble velocity variance to the square of the mean is $O(0.1)$. For these conditions Spelt \& Sangani predict that the homogeneous suspension will be unstable and clustering into horizontal rafts will take place. Evidence for bubble clustering is obtained by analysis of video images. The fluid velocity variance is larger than would be expected for a homogeneous suspension and the fluid velocity frequency spectrum indicates the presence of velocity fluctuations that are slow compared with the time for the passage of an individual bubble. These observations provide further evidence for bubble clustering.

\section{Introduction}

Hydrodynamic interactions and direct collisions between particles, drops, or bubbles can have a dramatic effect on particulate and multiphase flows. Through the comparison of careful experimental measurements with analytical theories and numerical simulations, considerable progress has been made in the understanding of suspensions in which viscous forces dominate on the particle length scale. The current understanding of the effects of particle interactions on inertial suspensions is much more limited. One type of inertial suspension, for which extensive theoretical work has been conducted, is a suspension of spherical, high-Reynolds-number bubbles. However, there is a dearth of experimental measurements with which these theories can be compared. 
The purpose of this paper is to present measurements of the flow properties of a nearly monodisperse suspension of millimetre sized bubbles rising through water in a vertical channel. This system approximates the ideal conditions of high Reynolds number and low Weber number that has been treated theoretically. We will also discuss the development of measurement protocols for suspensions of millimetre size bubbles.

The study of suspensions in which the inertia of the phases has a significant effect is challenging and their treatment is, in general, more difficult than that of low-Reynolds-number suspensions. There is a special case of an inertial suspension that is particularly amenable to theoretical analysis: a suspension of surfactant-free, spherical, high-Reynolds-number bubbles. In this case, the vorticity produced by the bubble motion is small (Moore 1963) and the fluid velocity can be expressed as the gradient of a potential obtained from solving Laplace's equation. It is possible to derive, from first principles, equations of motion for this type of bubble suspension (Biesheuvel \& Gorissen 1990; Sangani \& Didwania 1993a; Zhang \& Prosperetti 1994; Bulthuis, Prosperetti \& Sangani 1995; Kang et al. 1997; Spelt \& Sangani 1998; Yurkovetsky \& Brady 1996). In addition, dynamic simulations of many interacting bubbles can be conducted (Smereka 1993; Sangani \& Didwania 1993b). The potential flow approximation is applicable in the limits of high bubble Reynolds number and low bubble Weber number. The Reynolds number is defined as $R e=\rho_{f} d_{b} u_{b} / \mu$, where $\rho_{f}$ is the fluid density, $d_{b}$ is the bubble diameter, $u_{b}$ is the relative velocity of the bubble with respect to the fluid and $\mu$ is the fluid viscosity. The Weber number is defined as $W e=\rho u_{b}^{2} d_{b} / \sigma$, where $\sigma$ is the surface tension of the liquid-gas interface. The application of potential flow theory also requires that Marangoni effects be negligible, so that the tangential stress at the gas-liquid interface is small. Comparison between measured rise velocities for gas bubbles in water and theoretical predictions indicate that the potential flow theory for spherical bubbles is reasonably accurate for bubble diameters of about $1 \mathrm{~mm}$ and the agreement between theory and experiment can be improved by including the effects of bubble deformation (Moore 1965; Duineveld 1995). Although the development of these theories represents a significant step towards the understanding of inertial suspensions, the extent of their applicability must be critically determined by comparison with experimental measurements.

There have been a large number of experimental studies that examine the behaviour of bubbly liquids since they are widely used in practical applications (Molerus \& Kurtin 1985); however, most of the previous studies deal with the behaviour of bubble columns in which the bubble size and polydispersity are neither small nor controlled. These studies generally do not satisfy the dual limit of small Weber and large Reynolds number for which the potential flow theories may be applicable; therefore, direct comparisons are not possible. For bubbles rising under the action of gravity, there exists a narrow range of bubble diameters for which this dual limit is satisfied. Bubbles of $1 \mathrm{~mm}$ in diameter rising in water move at approximately $30 \mathrm{~cm} \mathrm{~s}^{-1}$ in straight trajectories without shape oscillations and are close to spherical in shape.

Another feature that makes the previous experimental studies deviate from the ideal conditions for direct comparison with potential flow theories is the polydispersity of the bubbles which results, in part, from coalescence. In concentrated suspensions, coalescence often leads to a very wide distribution of bubble diameters that may evolve over time. Coalescence can be prevented by the addition of salt to the liquid phase. Salt gives rise to short-range repulsive forces (known as hydration forces) between bubble interfaces which prevent coalescence. Lessard \& Zieminski (1971) have shown that a sufficient concentration of salt in aqueous solution will prevent coalescence in bubble columns. Because the surface tension of the air-water interface 
is relatively insensitive to salt concentration, salt does not produce Marangoni effects (unlike organic surface active substances).

One previous study (Valukina, Koz'menko \& Kashinskii 1979) used salt to prevent coalescence and thereby obtained a suspension of bubbles with diameters of $0.5-1 \mathrm{~mm}$. In that study, the radial volume fraction profile was measured in an upward vertical pipe flow for dilute suspensions. In such a flow, lift forces drive bubbles towards the wall, while shear-induced bubble-bubble collisions may push bubbles toward the centre of the pipe. The parameter regime explored by Valukina et al. was limited and in many of the experiments the majority of the bubbles were concentrated in a layer that was about 1.5 bubble diameters thick near the tube wall. This makes it unlikely that the measurements can be compared with a continuum description of the suspension. Lammers \& Biesheuvel (1996) performed experiments in a bubble column in which the bubble size variation was small to study the stability of concentration waves. With their experimental setup the bubble size variation was controlled; however, the system was not able to generate small enough bubbles to fall within the small Weber number and large Reynolds number regime.

The experiment presented in this paper was designed to study a bubble suspension which fulfilled as closely as possible the strict requirements for a direct comparison with the theories for bubble suspensions with potential flow interactions. While the potential flow approximation has been shown to give a reasonably accurate description of the rise of single bubbles (Moore 1965; Duineveld 1995), this study is the first to critically examine its applicability to a suspension of bubbles rising due to buoyancy. Direct comparisons between theoretical and experimental results for the decrease of the mean bubble velocity with gas volume fraction are obtained. The discrepancies found are analysed and attributed to features that appear in the experiment and are not accounted for in the currently available theories. In the experiment bubbles are slightly oblate and their aspect ratio changes as the gas volume fraction increases. The containing walls are found to have a significant effect, as bubbles experience a decrease of velocity as a result of bubble-wall collisions. Also, bubble-bubble and bubble-wall collisions cause bubbles to deform, introducing energy dissipation mechanisms not considered in current theories.

A distinctive feature that has been observed in the models and simulations of bubbly liquids in potential flow is the appearance of horizontally oriented clusters of bubbles (Smereka 1993; Sangani \& Didwania 1993b). Bubble suspension images and the spectrum of the liquid velocity produced by the bubbles are examined to provide evidence concerning the extent of bubble clustering in our suspensions.

Other mechanisms of clustering are known for bubbly liquids. Lammers \& Biesheuvel (1996) observed clustering of bubble clouds as a result of concentration instabilities that arise at sufficiently large bubble volume fractions due to the dependence of the drag coefficient on bubble concentration. Lin et al. (1996) observed clustering and swirling bubble structures resulting from polydispersity of the bubble size. This is the first experimental study showing horizontal clustering due to potential flow for dilute, monodisperse systems.

This paper presents the experimental results obtained for a suspension of bubbles rising in water for which the Reynolds number is large, $O(100)$, and the Weber number is small, $O(1)$. The device to generate the bubbles was designed to produce suspensions with bubbles of approximately $1 \mathrm{~mm}$ in diameter and with small polydispersity. An impedance probe, a hot-wire probe and video image processing were used to obtain measurements of the bubble size and shape, bubble velocity, bubble velocity variance, fluid velocity variance and bubble collision rate as functions of the mean bubble 


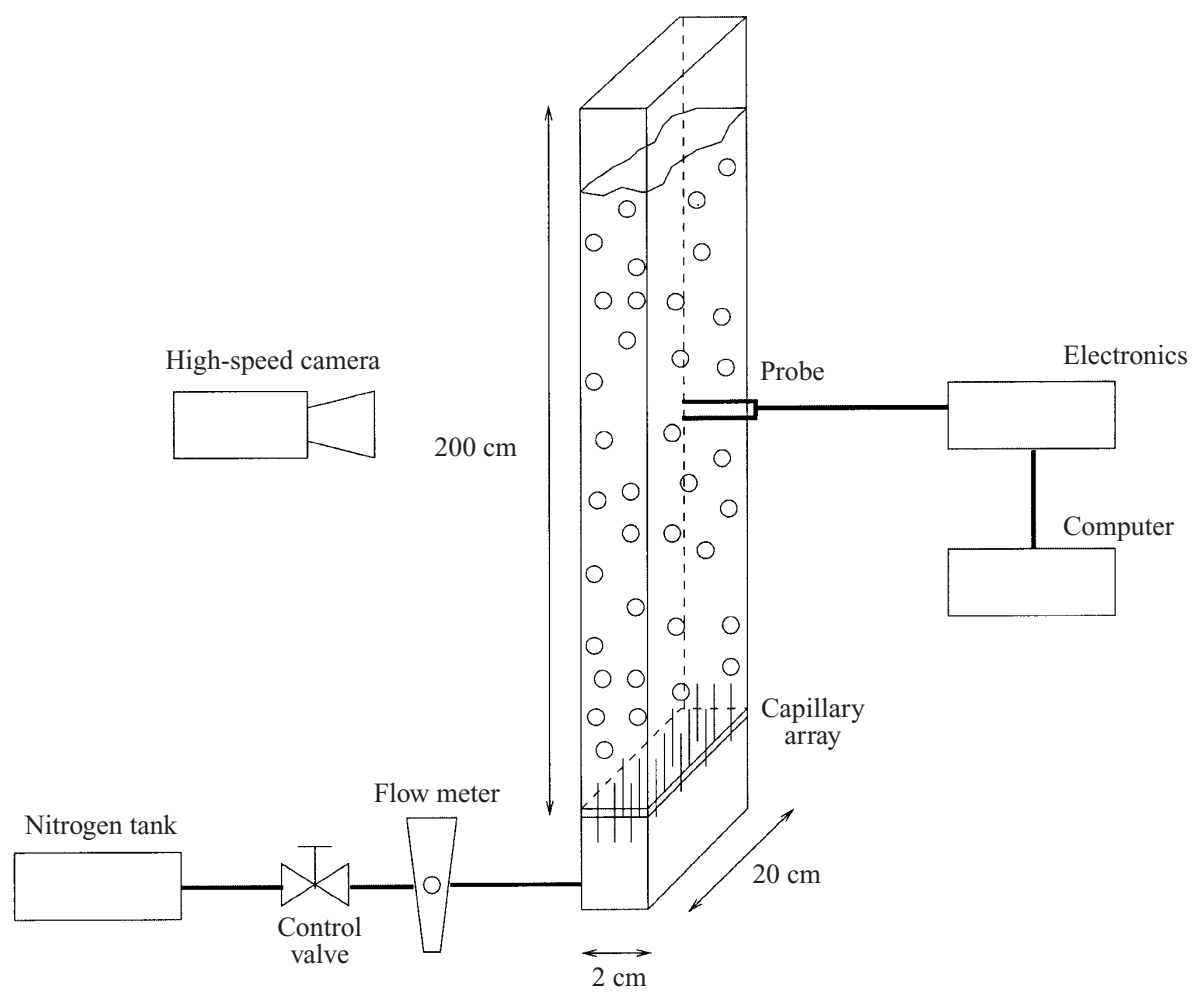

FIGURE 1. Experimental setup.

concentration. A detailed description of the instrumentation is provided. The results show that the mean bubble velocity decreases as the bubble concentration increases, at a rate comparable to that predicted by Spelt \& Sangani (1998). The fluid and bubble velocity variances are observed to increase with gas volume fraction.

In a companion paper, Zenit, Koch \& Sangani (2000) present measurements of the gas volume fraction and liquid and bubble velocity profiles for a gravity-driven shear flow, in which the shear flow is generated by inclining the test channel a small angle with respect to the vertical.

\section{Experimental setup}

The experiment is shown schematically in figure 1. The Plexiglas cell has a thickness of $2 \mathrm{~cm}$ and a width of $20 \mathrm{~cm}$. The thickness is chosen to obtain a near-continuum suspension behaviour. The width is chosen so that the flow in the centre of the channel is nearly two-dimensional. The cell height of $200 \mathrm{~cm}$ is large enough for the velocity and volume fraction profiles to be fully developed. The channel is vertically aligned within $0.1^{\circ}$ using a digital level.

Nitrogen gas is introduced at the base of the channel through an array of capillaries. The capillaries are small enough to generate bubbles on the order of $1 \mathrm{~mm}$ in diameter, which satisfy the dual limit of small Weber number and large Reynolds number. The liquid phase is a dilute aqueous electrolyte solution $\left(0.05 \mathrm{moll}^{-1} \mathrm{MgSO}_{4}\right)$. The electrolyte introduces a short-range hydration repulsion force between two gas-liquid interfaces which inhibits bubble-bubble coalescence (Tsao \& Koch 1994). Magnesium 
sulphate $\left(\mathrm{MgSO}_{4}\right)$ was used because at very low concentrations it readily prevents coalescence (Lessard \& Zieminski 1971). The addition of this small amount of salt does not change the viscosity or density of the liquid or the surface tension of the gas-liquid interface significantly.

The mean gas volume fraction, $\alpha$, or hold up, is calculated by measuring the increase in liquid level after the bubbles are introduced to the cell,

$$
\alpha=\left(H_{o} / \Delta H+1\right)^{-1}
$$

where $H_{o}$ is the initial liquid level, and $\Delta H$ is the liquid level increase.

High-speed video photographs and digital image processing are used to obtain measurements of the bubble size and aspect ratio. Some measurements of the bubble velocity are obtained using this technique but these are limited to the case of dilute suspensions due to the opaque nature of bubbly fluids. Two types of probe are employed to characterize the flow of the bubble suspension. A commercial hot-film anemometer is used to measure the liquid velocity and the bubble-probe collision rate. A dual impedance probe was designed to measure the gas volume fraction, mean and fluctuating bubble velocities, the mean bubble chord length, and the bubble-probe collision rate. In this paper only bubble velocity results are presented.

\subsection{Bubble generation}

A device to produce the bubbly mixture was designed to keep the polydispersity of bubble diameter to the minimum possible. Capillaries with an inner diameter of $100 \mu \mathrm{m}$ were embedded in a perforated Plexiglas plate. Nearly 900 of these $65 \mathrm{~mm}$ long tubes were positioned in a hexagonal array, in order to achieve the maximum number of tubes per unit of area ( 28 capillaries per square $\mathrm{cm}$ ) without interference between neighbouring capillaries. With this arrangement, the gas flow rate per tube was small and the pressure drop across each tube was large. These two factors allowed the bubble formation to occur in a quasi-steady fashion, resulting in the production of uniform size bubbles (Og̃uz \& Prosperetti 1993). The flow rate per capillary used in our experiments was always small enough so that the bubble size could be expected to be independent of flow rate according to Og̃uz \& Prosperetti's criterion.

Figure 2 shows pictures of the bubbles produced for three typical mean gas concentrations. The images were obtained by photographing the suspension through the $20 \mathrm{~cm}$ wall of the channel (the long dimension). The nearly monodisperse nature of the mixtures can be clearly observed. The mean bubble diameter is approximately $1.4 \mathrm{~mm}$. Also, some evidence of the clustering in bubble suspensions predicted by Spelt \& Sangani (1999), Sangani \& Didwania (1993) and Smereka (1993) can be observed. More quantitative evidence for bubble clustering will be discussed in the experimental results section.

The bubble size and aspect ratio were measured using digital image processing. The results are presented in the next section. The measurement error resulting from the finite pixel size of the digital images was calculated to be on the order of $3 \%$.

\subsection{Hot-film anemometry}

A hot-film anemometer is used to measure the bubble-probe collision rate and the liquid fluctuating velocity in the bubble suspension. A sketch of the probe geometry is shown in figure 3. The hot-film probe used in the experiments consists of a $25 \mu \mathrm{m}$ diameter platinum wire coated with quartz to provide electrical insulation from the conducting liquid. The length of the sensitive part of the wire is $350 \mu \mathrm{m}$. The active wire is attached to the probe supports which are oriented perpendicular to the mean 
(a)

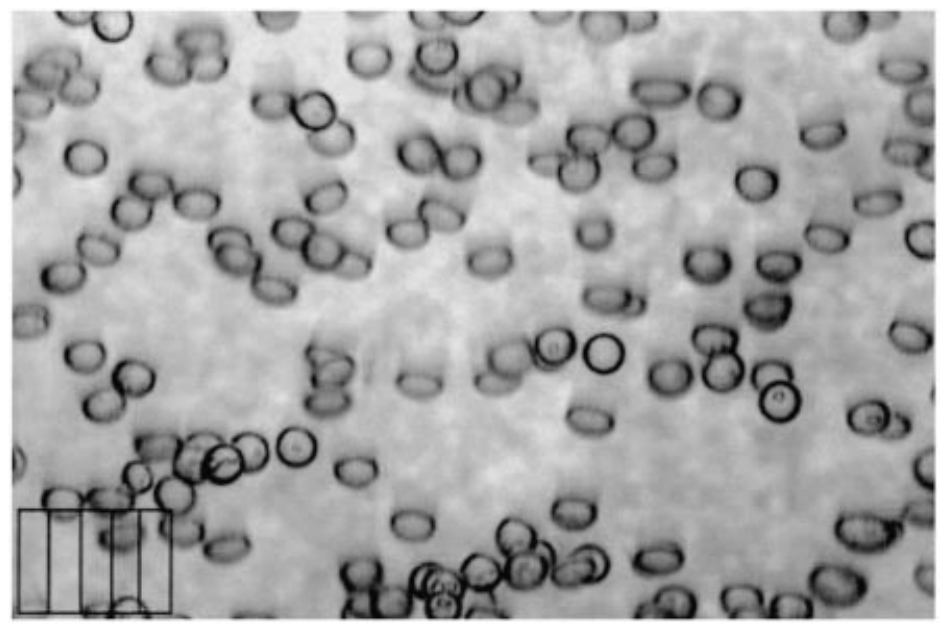

(b)

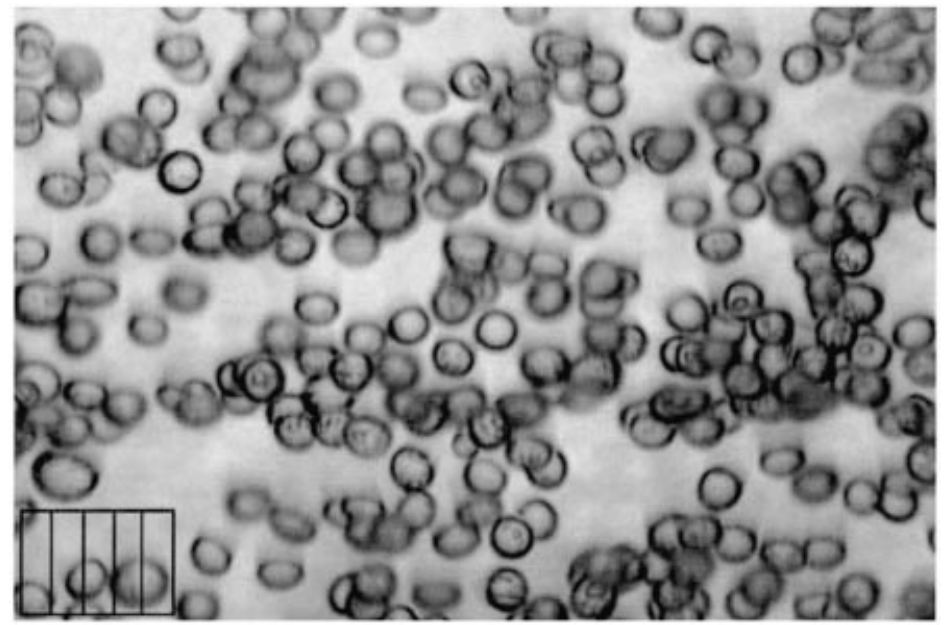

(c)

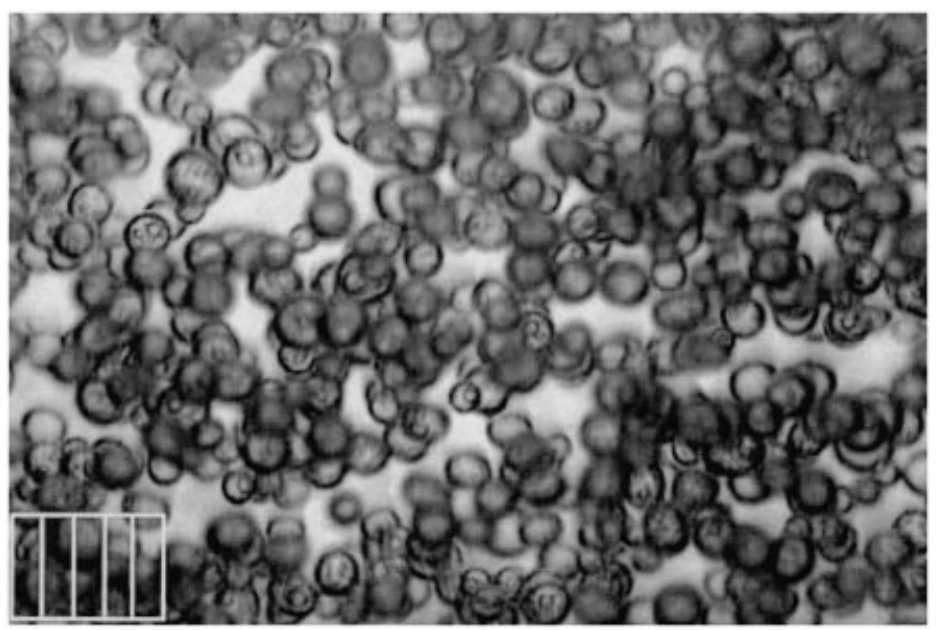

Figure 2. Bubble size and distribution for typical mean gas volume fractions. The spacing in the grid is $1 \mathrm{~mm}$. (a) $\alpha=0.02,(b) \alpha=0.05,(c) \alpha=0.10$. 


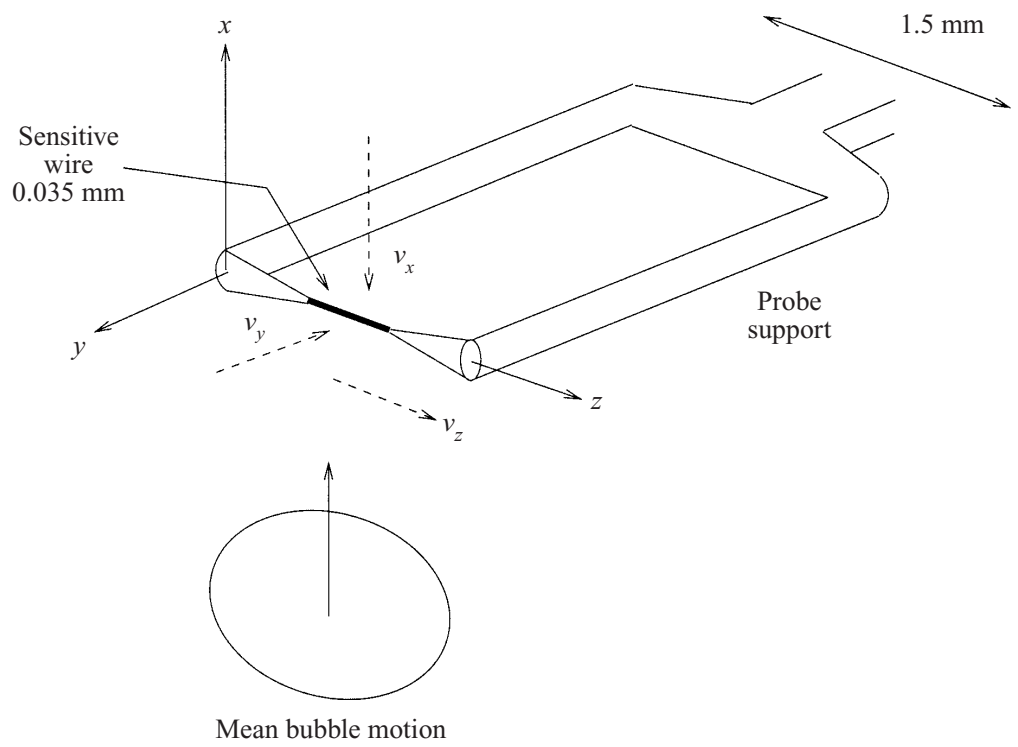

FIGURE 3. Sketch of the hot-wire probe and its orientation with respect to the mean bubble motion.
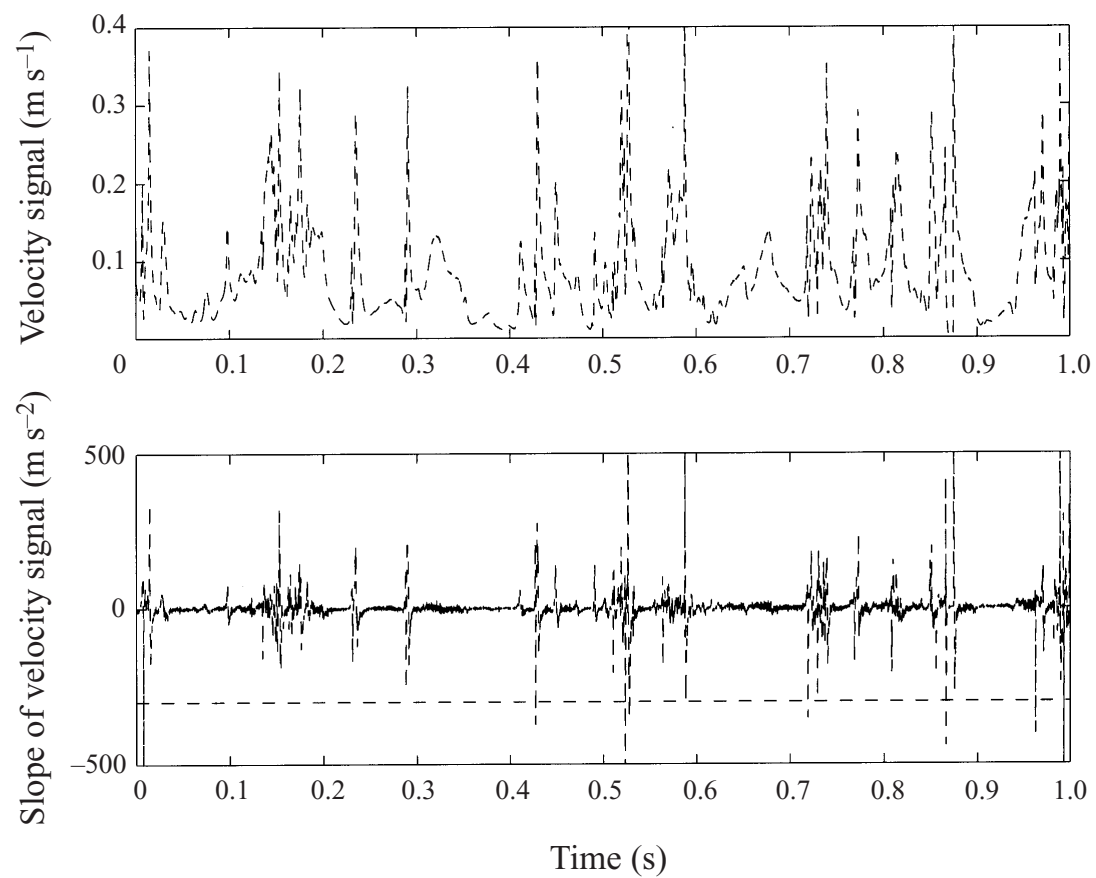

FIGURE 4. Typical fluid velocity signal and fluid velocity signal slope for a mean gas volume fraction of 0.05 . The dashed line denotes the collision detection threshold level.

bubble motion to ensure that the same signal is detected for positive and negative vertical velocities. A typical velocity signal obtained from the hot-wire anemometer immersed in the bubble suspension is shown in figure 4 along with the time derivative of the velocity signal.

Hot-film probes have been used before for measurements of two-phase slug and 
bubbly flows (Brunn 1995). However, most studies involved very high-speed flows with large bubble diameters. During the interaction of a large bubble with the probe at a high velocity, the probe is thought to pierce the bubble interface. In this study, using high-speed photography, it has been observed that small bubbles $(1-2 \mathrm{~mm})$ moving at speeds comparable to their terminal velocity are not pierced by the anemometer probe. Instead, the bubbles bounce off the probe. By synchronizing the velocity data acquisition system with the high-speed camera, it was possible to observe that when a bubble collides with the sensitive part of the hot wire a sharp decrease of the slope of the fluid velocity signal occurred. By analysing the sudden change in the signal slope when moving bubbles made contact with the hot wire, collisions were detected and counted. An appropriate threshold level for bubble collision was determined by performing experiments in which a single bubble was released at a series of different horizontal positions relative to the probe and the signal slope that occurred when a bubble collided with the probe was noted. The minimum slope observed was different for direct collisions, grazing collisions and near-misses of the bubbles and the probe. By this procedure the cross-sectional area for bubble detection was measured to be $1.803 \mathrm{~mm}^{2}$ for a bubble detection threshold level of $-300 \mathrm{~m} \mathrm{~s}^{-2}$. A slight change in area with threshold level occurs due to grazing collisions; however, it is clear that $-300 \mathrm{~m} \mathrm{~s}^{-2}$ corresponds to a cut-off between direct hits and misses. Figure 4 shows the collision detection level used in the experiments reported here as the dashed line.

The hot-wire anemometer responds primarily to velocity components perpendicular to the axis of the wire. The velocity signal obtained when the wire is parallel to an imposed velocity is only $20 \%$ of that obtained when the wire is perpendicular to the flow. For simplicity, we will assume that the anemometer is measuring the projection of the velocity vector into the plane perpendicular the wire axis. Owing to the symmetry of the probe, the heat transfer from the wire is equal for upward and downward flows relative to a horizontally oriented hot wire. Thus, the anemometer measures the magnitude $\sqrt{v_{x}^{2}+v_{z}^{2}}$ of the fluid velocity perpendicular to the wire $(y)$ axis. Therefore the average of the fluid velocity signal illustrated in figure 4 is non-zero even though the mean velocity is zero in a vertical channel. The velocity signal provides a measure of the fluctuations. By taking measurements with the hot wire oriented vertically and horizontally, the variances of both the horizontal and vertical velocities can be determined.

\subsection{Dual impedance probe}

Impedance probes have been used widely by the multi-phase flow community to measure gas volume fraction (Ceccio \& Georges 1996). However, in most cases such techniques are used to obtain a spatial average of the gas volume fraction across the thin dimension of the flow apparatus. The technique presented here can identify single bubbles interacting with the measuring probe in a localized small measuring volume. This characteristic of the measuring system is needed to measure the spatial variation of the bubble concentration and velocity across the thin direction as will occur in our future investigation of inclined channel flows (Zenit et al. 2000). Impedance systems able to measure and detect individual bubbles have been used only by a few investigators (Waniewski 1999; Liu \& Bankoff 1993).

The system can detect local changes of electrical impedance in bubbly mixtures. It uses the difference in electrical impedance between the gas and liquid phases to determine the residence time of bubbles in a small measuring volume adjacent to the probe tip. The probe arrangement is shown schematically in figure 5. An electrode is embedded in a thin hypodermic needle, which acts as the ground. The electrode 


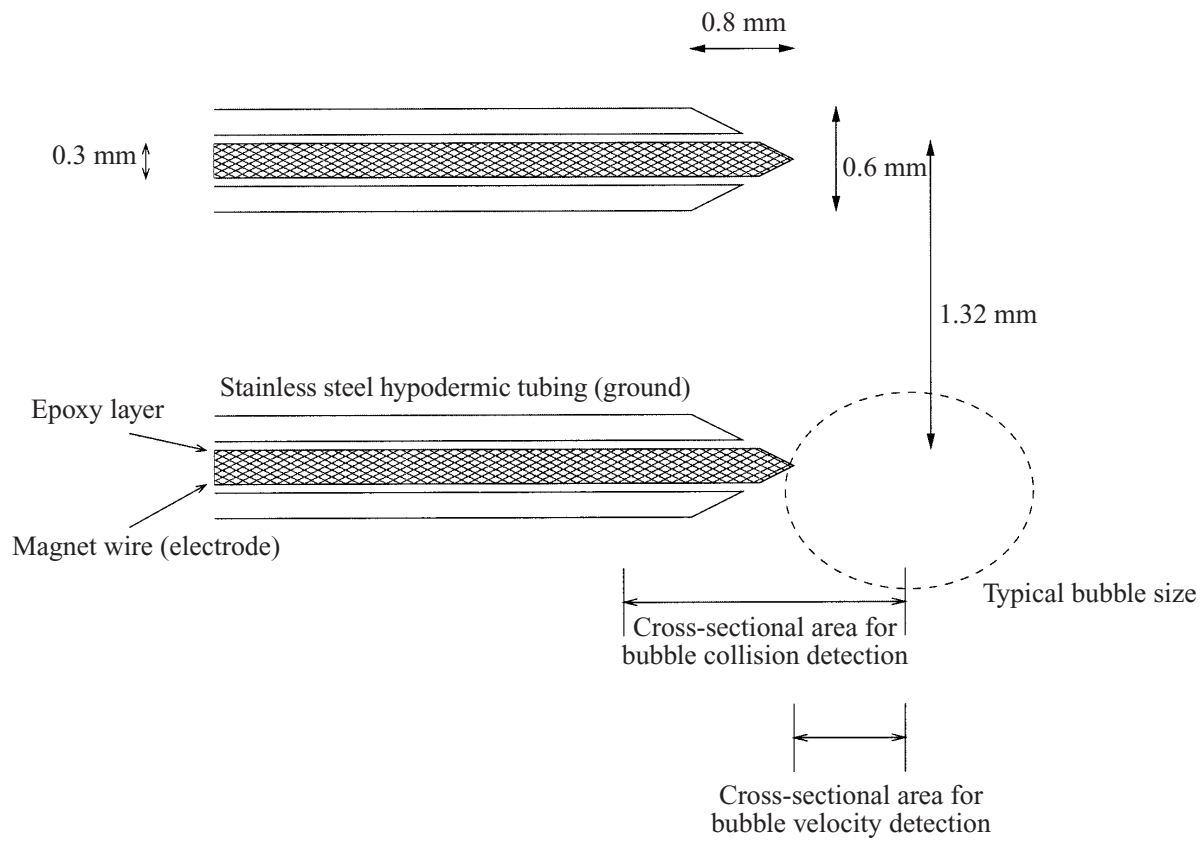

FIGURE 5. Sketch of the dual impedance probe. The figure is drawn to scale.

carries a rapidly oscillating voltage. When bubbles pass near the tip of this probe, the local impedance and the current through the electrode are affected. The amplitude of the current signal is then converted to a DC voltage signal by an electronic circuit. The probes and electronic circuits used here are based on the design proposed by Waniewski (1999). A typical signal change detecting an individual bubble as it passes near the tip of the probe is shown in figure 6.

In a vertical channel, the gas volume fraction can be determined simply by measuring the holdup (equation (2.1)). It is possible to measure the local gas volume fraction by establishing a voltage threshold level that discerns whether or not there is a bubble in the vicinity of the tip of the probe. The vertical channel provides a convenient setting in which to validate a volume-fraction measurement technique that can be used in more complicated situations where the volume fraction varies with position. A detailed analysis of the performance of the impedance probe to measure local gas volume fraction is presented in the companion paper (Zenit et al. 2000). For this paper the gas volume fraction is obtained from the column holdup.

A second identical probe is positioned at a known distance above the leading probe. If a bubble passes near both probes, the signals produced in each of them will be similar but shifted in time, as shown in figure 6 . The signals are cross-correlated and the delay time, $\tau_{\max }$, can be accurately calculated as the value of signal shift time $\tau$ that maximizes the cross-correlation function, $F_{V_{1} V_{2}}$,

$$
F_{V_{1} V_{2}}(\tau)=\frac{1}{t_{s}} \int_{0}^{t_{s}} V_{1}(t) V_{2}(t-\tau) \mathrm{d} t
$$

where $t_{s}$ is sampling time and $V_{1}$ and $V_{2}$ are the voltages obtained from the leading and trailing probes respectively. The bubble velocity can be obtained as

$$
u_{b}=\frac{D}{\tau_{\max }}
$$




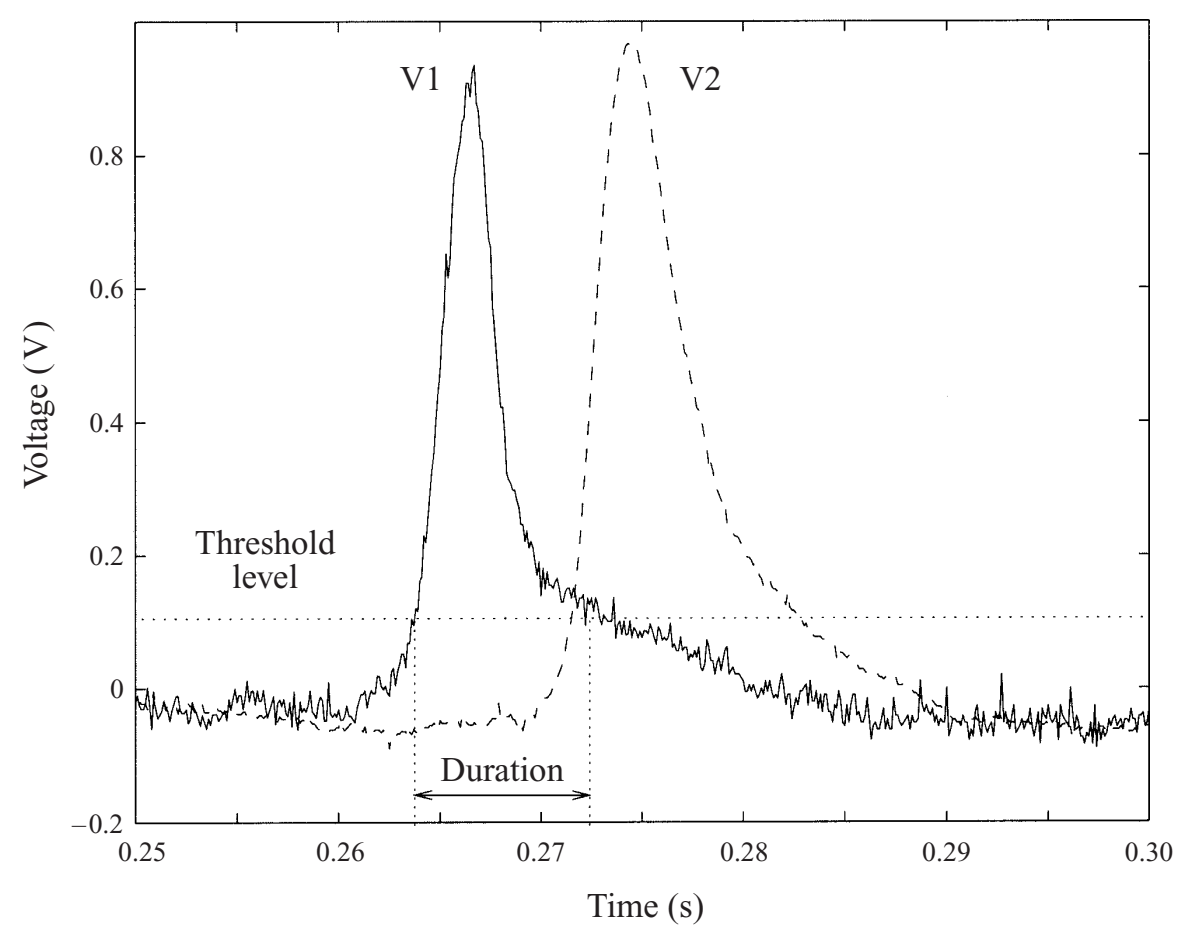

FIGURE 6. Typical voltage signal obtained from the impedance measurement resulting from a bubble passing near the tip of the probe. The solid line denotes the signal obtained from the leading probe, the dashed line shows the signal from the trailing probe. The dotted line denotes the voltage threshold level.

where $D$ is the separation distance between probes. The velocity calculated in this manner was compared with the velocity obtained using video image processing. The results differ only by $1 \%$ for a $1.3 \mathrm{~mm}$ bubble moving at $27 \mathrm{~cm} \mathrm{~s}^{-1}$. Note that for this technique to be appropriate the bubble velocity must be nearly unidirectional, which is true for the flows studied in this paper. The bubble velocity calculated from the cross-correlation function uses the entire time series (typically $100 \mathrm{~s}$ ) from the impedance probe. The resulting measurement contains information from many different bubble events.

Information concerning the probability distribution of bubble velocities can be obtained by further processing of the signals. A software program searches and identifies voltage pulses corresponding to bubble detections by the leading probe, i.e. events for which the signal rises above the voltage threshold of $0.2 \mathrm{~V}$. To determine if a similar pulse, shifted in time, was produced in the trailing probe, a local crosscorrelation function is performed. A discriminating criterion is adopted to eliminate erroneous signals. If the calculated velocity is improbable, it is assumed that the signals in the two probes were caused by two different bubbles and, therefore, the velocity is discarded. The algorithm discards velocities that are more than $50 \%$ larger than the terminal velocity and smaller than a tenth of the terminal velocity. In addition, events that yield small values of the maximum in the cross-correlation function, $F_{V_{1} V_{2}}<0.0015 \mathrm{~V}^{2}$, are discarded. The sample length used for the determination of the local cross-correlation function is $20 \mathrm{~ms}$. In this way a set of individual velocity measurements is obtained, and therefore the variance of the vertical bubble velocity, $T_{b}$, can be determined. 
Different separations between the probes were tested. A distance of $1.32 \mathrm{~mm}$ was used for the experimental results presented here. Larger distances resulted in poor cross-correlation values and shorter distances resulted in interference between the electrical signals of the probes.

The calculations and the signal analysis are performed on the digitized voltages from the probes. The sampling rate used was $10 \mathrm{kHz}$ per probe, both for the impedance probes and the hot-wire system. The recording time per data point was, at least, $100 \mathrm{~s}$ for all cases. The initial liquid height, $H_{o}$, was $1.50 \mathrm{~m}$ and was kept constant for all experiments.

\section{Experiments}

With the instrumentation described above, precise measurements of the flow properties were obtained. Experiments were performed to determine the effect of gas volume fraction on the bubble collision rate, the mean and variance of the bubble velocity, and the fluid velocity variance. The range of gas volume fractions studied was from 0 to 0.20 . The size, size distribution and aspect ratio of the bubbles in the suspension were obtained as a function of gas volume fraction. Most of the measurements were taken $100 \mathrm{~cm}$ above the bubble injector. However, a series of experiments confirmed that the variation of the gas volume fraction with vertical position in the channel was small.

\subsection{Single bubble experiments}

A series of observations of single bubbles rising in a large pipe $(20 \mathrm{~cm}$ in diameter and $40 \mathrm{~cm}$ in length) was conducted to determine the terminal velocity of a single bubble of the same size as those produced in the channel. A single capillary, identical to those used in the bank of capillaries to generate the bubble suspension, was used to generate individual bubbles. Using video image processing, the terminal velocity of the bubble $u_{\infty}$ was found to be $0.320 \mathrm{~m} \mathrm{~s}^{-1} \pm 3 \%$ with an equivalent diameter equal to $1.346 \mathrm{~mm} \pm 3.5 \%$. This results in a terminal Reynolds number of 431 and a Weber number of 1.969 , assuming a value of $0.07 \mathrm{~N} \mathrm{~m}^{-1}$ for the surface tension and a value of $0.001 \mathrm{~Pa}$ s for the liquid viscosity. The aspect ratio of the bubble was $1.70 \pm 6 \%$. The motion of the bubble was rectilinear and no bubble shape or trajectory oscillations were observed. Based on Moore's (1965) calculations, the rise velocity of a bubble with the same equivalent diameter would be $0.317 \mathrm{~m} \mathrm{~s}^{-1}$, which is $0.9 \%$ lower than the experimentally measured velocity. The measurements of Duineveld (1995) show that for a bubble with the same equivalent diameter moving in pure water the terminal velocity is approximately $0.339 \mathrm{~m} \mathrm{~s}^{-1}, 5.9 \%$ higher than the present measurement. This discrepancy could be expected since in the present experiments the water was deionized and filtered but the precautions used to assure purity were not as stringent as in Duineveld's experiments. The measured aspect ratio is $11 \%$ higher than the value predicted by Moore (1965), and 13\% higher than the value measured by Duineveld (1995) for the same value of Weber number. This discrepancy may result from deviations of the actual surface tension from the assumed value and/or from the existence of a small amount of impurities in the liquid.

\subsection{Bubble size and aspect ratio}

The mean and standard deviation of the equivalent diameter obtained from the image analysis are shown in figure 7, as functions of the mean gas volume fraction $\alpha$. The 


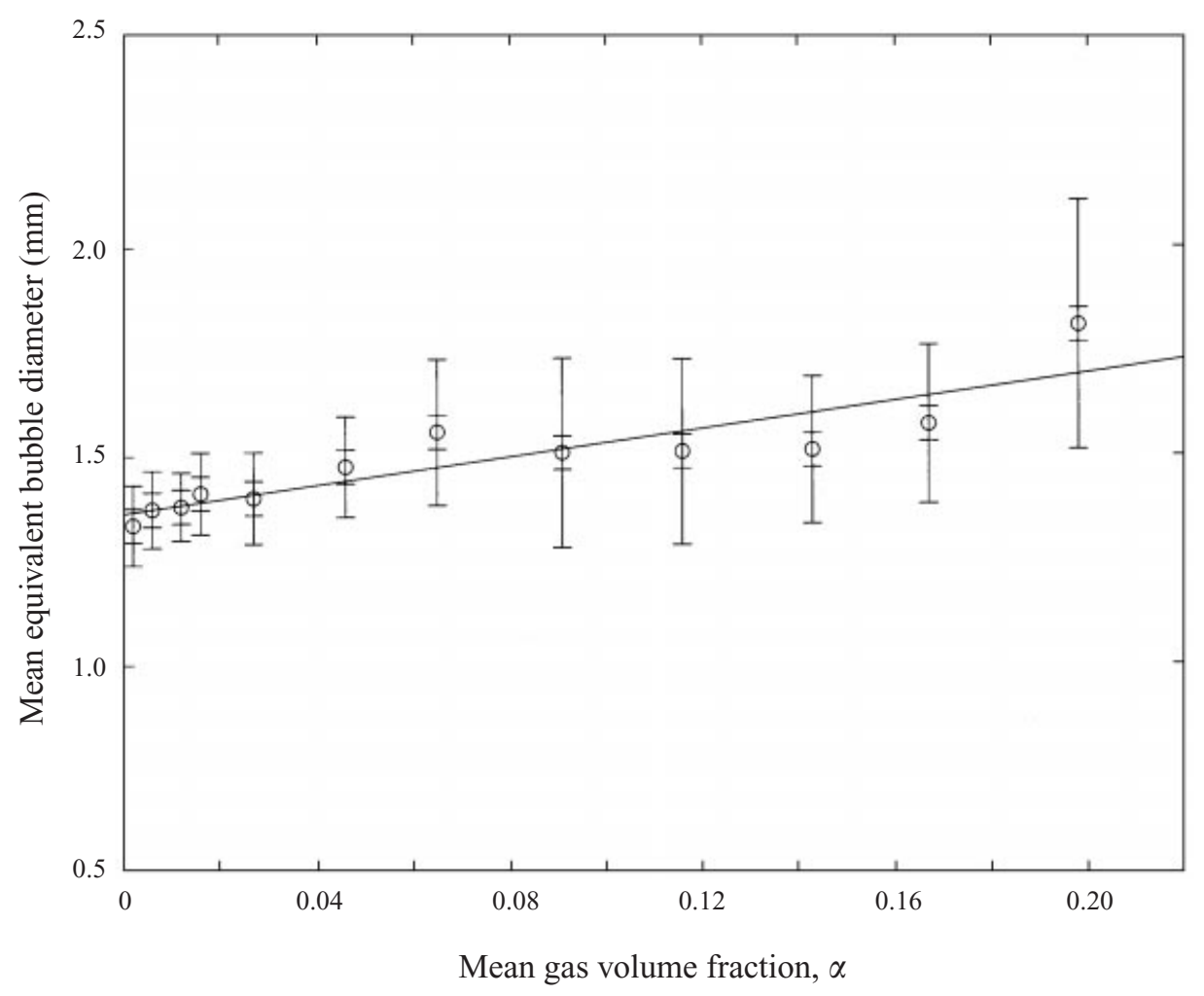

FIGURE 7. Equivalent bubble diameter, $d_{e q}$, as a function of mean gas volume fraction, $\alpha$. The short bars indicate the measurement error associated with the finite pixel size. The long bars indicate the bubble size standard deviation. The solid line depicts the linear fit shown in equation (3.2).

mean equivalent bubble diameter is calculated by

$$
d_{e q}=\left(d_{\text {long }}^{2} d_{\text {short }}\right)^{1 / 3}
$$

where $d_{\text {long }}$ is the long axis length of the oblate bubble and $d_{\text {short }}$ is the short axis length. For this measurement it is assumed that the bubble is axisymmetric with respect to its short axis direction. The mean bubble size was found to increase slightly for gas volume fractions up to 0.20 . The bubble size standard deviation, depicted by the long bars, was approximately $10 \%$ and constant for gas volume fractions smaller than 0.15 . Note that, although the increase in the size of the bubbles is small, it has a significant effect on the measurement of the gas volume fraction as discussed earlier. Coalescence of bubbles was not easily observable in the range of gas volume fractions studied here. However, the slight increase in bubble diameter may result from a small amount of coalescence or from small changes in the size of the bubbles produced by the capillaries with increasing gas flow rate. The variation of the equivalent bubble diameter with gas volume fraction can be approximated using the expression

$$
d_{e q}=1.66 \alpha+1.364
$$

where the units of $d_{e q}$ are $\mathrm{mm}$. The statistical error associated with the size measurement is on the order of $3 \%$.

Figure 8 shows the measurement of the bubble aspect ratio $\chi$ as a function of the mean gas volume fraction, $\alpha$. The aspect ratio is defined as $\chi=d_{\text {long }} / d_{\text {short }}$. Note that 


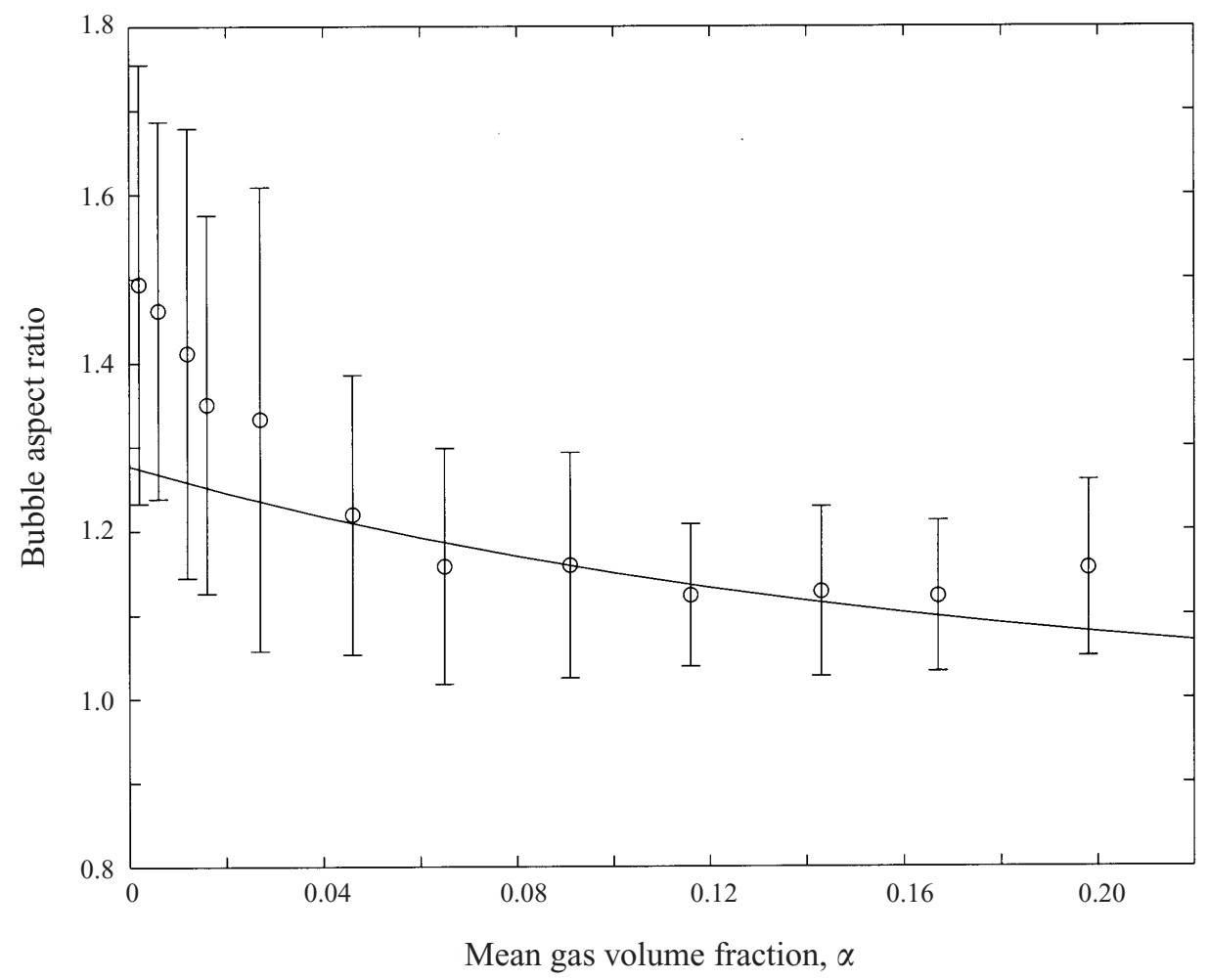

FiguRE 8. Bubble aspect ratio, $\chi$, as a function of mean gas volume fraction, $\alpha$. The bars indicate the bubble aspect ratio standard deviation. Measurements obtained $50 \mathrm{~cm}$ above the bubbler. The solid line presents the prediction from equation (3.3).

the shape of the bubbles in the suspension is closer to spherical as the gas volume fraction increases. This results in part from the reduction of the mean rise velocity as the bubble concentration increases. The experimental results on the reduction of the mean terminal velocity are presented later in this section. Moore (1965) proposed a model to predict the bubble aspect ratio for slightly deformed bubbles moving in potential flow, valid for moderate values of the Weber number in the absence of bubble-bubble interactions. The implicit equation for the aspect ratio as a function of the Weber number is

$$
W e(\chi)=4 \chi^{-4 / 3}\left(\chi^{3}+\chi-2\right)\left[\chi^{2} \sec ^{-1} \chi-\left(\chi^{2}-1\right)^{1 / 2}\right]^{2}\left(\chi^{2}-1\right)^{-3} .
$$

Since the Weber number can be calculated from the present experiments as a function of the bubble volume fraction, the change in bubble aspect ratio is obtained as a function of gas volume fraction. The solid line in the plot shows the predicted aspect ratio from equation (3.3). The bubble size change with gas volume fraction is taken from equation (3.2) and the decrease in bubble velocity is obtained from equation (3.4). The expression proposed by Moore does predict a decrease in the bubble aspect ratio; however it underestimates the rapid decrease of the aspect ratio observed for gas volume fractions smaller than 0.05 . This difference may arise as a result of the hydrodynamic interactions and collisions of bubbles with one another and with the walls. Note also that the aspect ratio for a single bubble, $\chi_{o}=1.7$, presented in the previous section, is higher than the measured aspect ratio even in very dilute suspensions. 


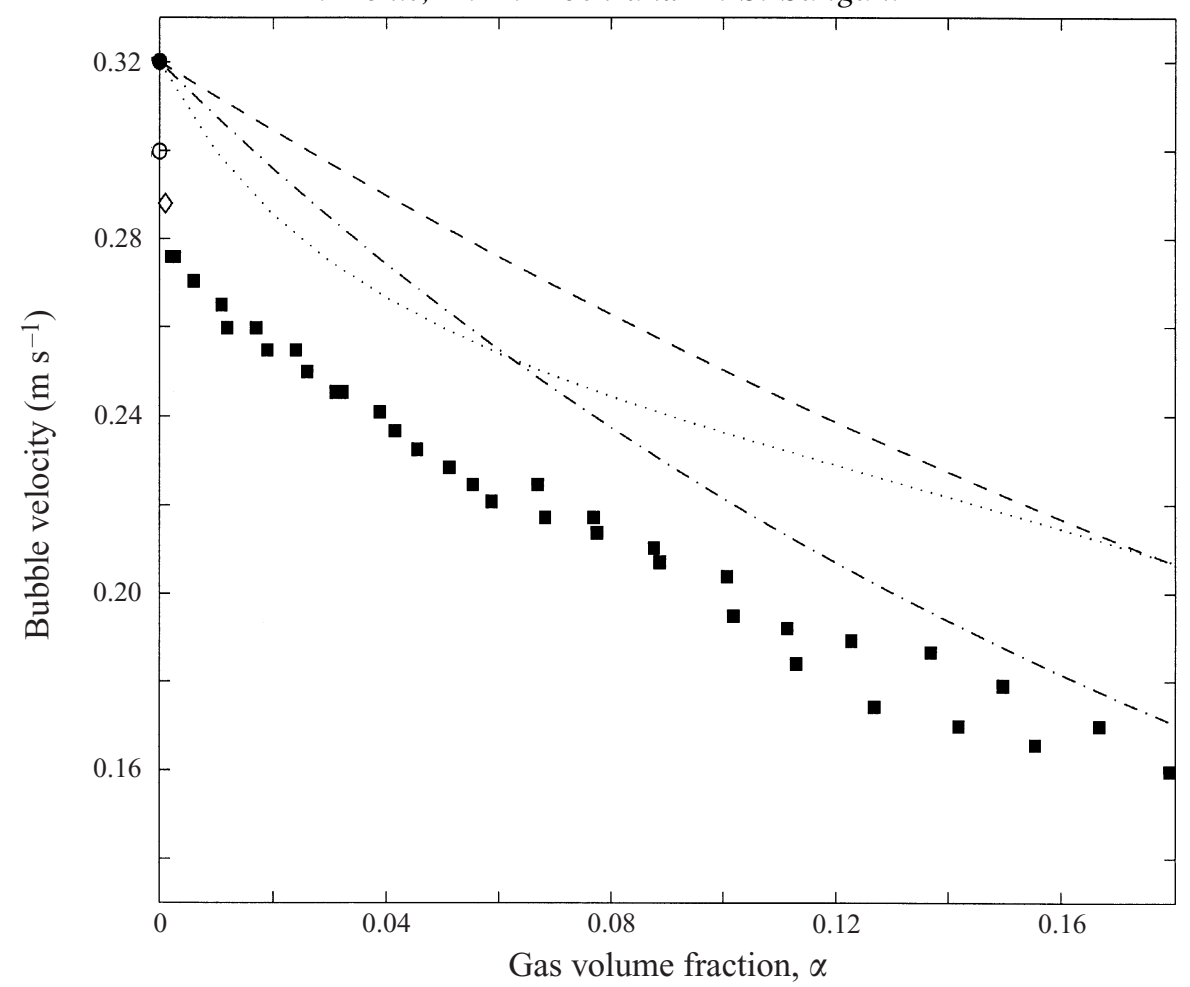

FIGURE 9. Bubble velocity as a function of mean gas volume fraction. The solid squares show the measurements using the dual impedance probe. The lines show the predictions from Spelt \& Sangani (1998) using $u_{\infty}=0.320 \mathrm{~m} \mathrm{~s}^{-1}$ and different values of the parameter $A:--, A=10 ;-\cdot-, A=20$; $\cdots, A=A(\alpha)$ from experiments shown in figure 10. The filled circle shows the terminal velocity of a bubble moving in a large channel $\left(u_{\infty}=0.320 \mathrm{~m} \mathrm{~s}^{-1}\right)$. The empty circle shows the velocity measured for a single bubble in the experimental channel. The diamond shows the measurement for a very dilute suspension in the channel.

\subsection{Bubble velocity and velocity variance}

The measurements of the bubble velocity were obtained by placing the probe in the centre of the channel where the velocity is nearly uniform. Measurements of the bubble velocity and bubble and fluid velocity variances were obtained for various gas volume fractions. It was found that all the profiles are uniform across the channel width and changes in the profiles were only observed near the walls (Zenit et al. 2000). Figure 9 shows the mean bubble velocity as a function of the gas volume fraction calculated from the column holdup. The mean bubble velocity decreases as the bubble concentration increases.

From the measurement of the bubble diameter (equation (3.2)) and the mean bubble velocities (equation (3.4)) an experimental measure of the Reynolds and Weber numbers can be obtained. The Reynolds number is observed to decrease monotonically with gas volume fraction from 380 for a dilute suspension to 260 for 0.20 gas volume fraction. The Weber number also decreases with the gas volume fraction from 1.5 to 0.5 for the same range of gas volume fraction. The dual limit of large Reynolds number and small Weber number is therefore satisfied for this range of concentrations.

The bubble velocity measurements can be fitted approximately to the expression

$$
u_{b}=u_{\infty}(1-\alpha)^{n}
$$


where $n$ is an experimentally determined parameter and $u_{\infty}$ is the bubble velocity for the limit of $\alpha \rightarrow 0$. The fit of the experiments results in a value of $n=2.796$ and $u_{\infty}=0.269 \mathrm{~m} \mathrm{~s}^{-1}$. It is important to note that the value of $n$ is larger than previously reported values for suspensions with comparable Reynolds numbers. Richardson \& Zaki (1954) report a value of $n=2.5$ for solid particles with a Reynolds number of approximately 250 and Ishii \& Zuber (1979) report a value of $n=2.0$ for drops and bubbles with the same range of Reynolds numbers. The reasons for this strong hindering behaviour are discussed below in $\$ 3.3 .2$. Note also that the inferred value of $u_{\infty}$ differs from the value measured for an individual bubble moving in a large pipe. Van Wijngaarden \& Kapteyn (1990) observed a similar phenomenon for bubbles of approximately twice the diameter of the bubbles in this study. To investigate the cause of this apparent discrepancy, additional experiments were performed to study the motion of individual bubbles in the test section.

For these experiments the bank of capillaries was replaced by an individual capillary where a single bubble could be formed. It was observed that the bubble moved at first in a straight trajectory, but after a certain distance (approximately $20 \mathrm{~cm}$ ), it began to experience trajectory oscillations. The bubble moved diagonally from wall to wall, with a trajectory that differed slightly from the vertical direction. The bubbles translated approximately three times the channel width in the vertical direction while moving from wall to wall. When the bubble came within one bubble diameter of the wall, it slowed its vertical velocity slightly, rotated, changed the direction of its horizontal translation, and moved toward the opposite wall. This behaviour is depicted schematically in figure 11 below. This translation from wall to wall resulted in a reduction of the mean bubble velocity as compared with that of a bubble of the same diameter moving in a large container. The mean velocity for this case was $0.298 \mathrm{~m} \mathrm{~s}^{-1}$, and is shown in figure 9 denoted by the circle on the $\alpha=0$ axis. This velocity, although smaller than the terminal bubble velocity, $u_{\infty}=0.320 \mathrm{~m} \mathrm{~s}^{-1}$, is still larger than that inferred from the fit of the velocity measurements obtained with the impedance probe. Note that in the case of a bubble moving in a large container no bubble trajectory oscillations were observed.

The mechanism behind this trajectory oscillation and how the bubble is repelled from the wall is not fully understood. A spherical bubble translating parallel to a wall experiences an attraction towards the wall, resulting from the interaction with its image (its potential flow image to satisfy the boundary condition at the wall). We observed that oblate bubbles are first attracted to and then repelled by the wall. This behaviour may result from the deformation of the bubble due to its interaction with its image. Duineveld (1994) performed experiments with interacting pairs of identical bubbles rising side-by-side due to buoyancy and observed a similar phenomenon for large oblate bubbles. In that study two identical bubbles with equivalent diameters smaller than $1.8 \mathrm{~mm}$ were observed to attract each other and interact repeatedly as they moved. For bubbles with diameters larger than $2.04 \mathrm{~mm}$ the oblate bubbles were observed to interact initially and then separate permanently.

Figure 10 shows the measured bubble vertical velocity variance $T_{b}$ normalized by the square of the mean bubble velocity, $u_{b}^{2}$, as a function of the gas volume fraction. The normalized variance increases with bubble concentration because the increasingly prevalent bubble-bubble hydrodynamic and collisional interactions increase the bubble velocity variance and decrease the mean of the bubble velocity. Along with the experimental data, the lines show the values used for the comparisons with the predictions of Spelt \& Sangani in figure 9. The parameter $A$ is the inverse of the normalized bubble velocity variance which, for the comparison with the experimental 


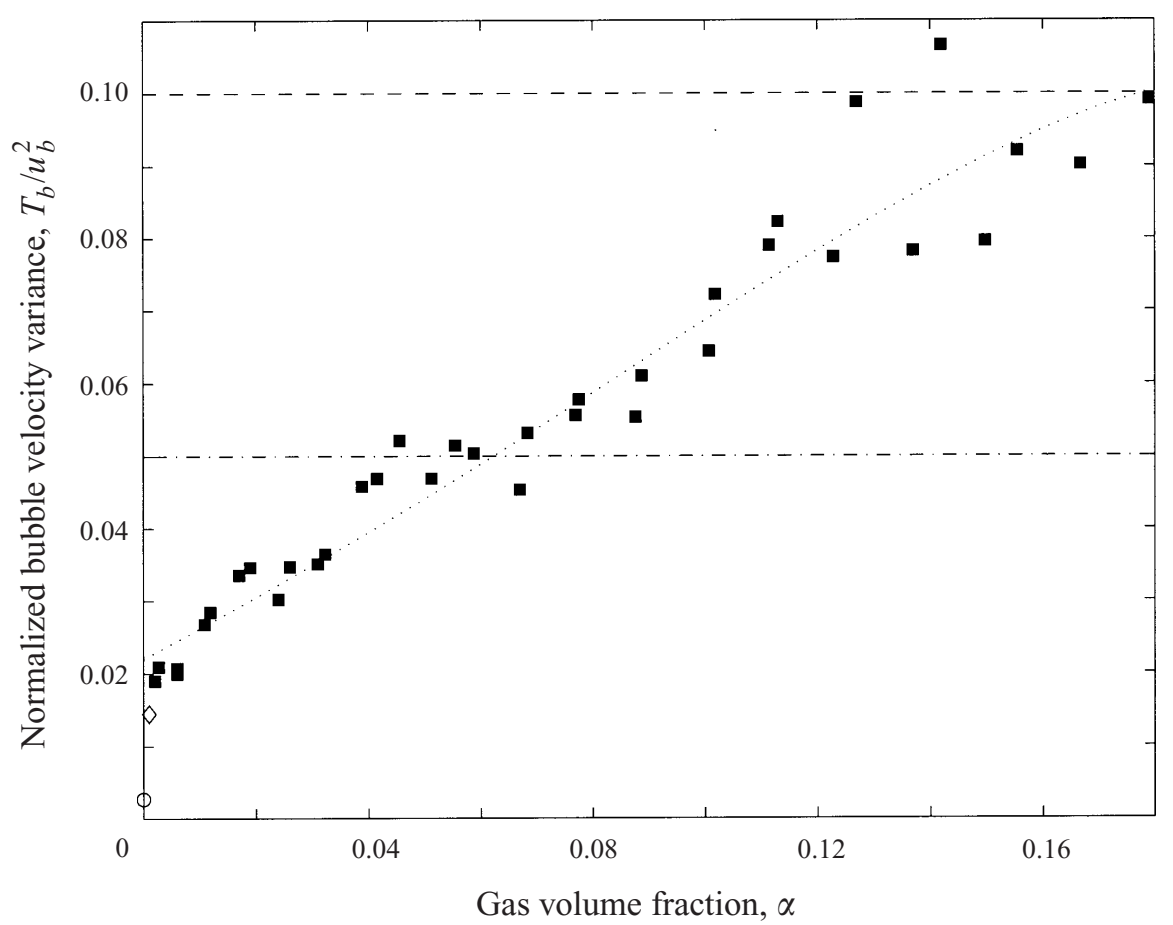

FIGURE 10. Normalized bubble vertical velocity variance as a function of mean gas volume fraction. The solid squares show the measurements using the dual impedance probe. The dashed and dash-dot lines represent the values of $A$ used for the bubble velocity predictions for Spelt \& Sangani (1998) in figure 9 ; the dotted line is the fit to the measurements. The circle shows the bubble velocity variance measured for a single bubble in the channel. The diamond represents the bubble velocity variance measured in a very dilute suspension.

measurements, only accounts for the vertical velocity variance. The dotted line shows the fit to the experimental data. It is important to note that the variance does not approach zero as the gas volume fraction tends to zero. From single bubble experiments in the channel, it was observed that bubbles underwent oscillating trajectories resulting from the bubble-wall interactions causing a decrease in the mean bubble velocity and a bubble velocity variance. For a single bubble, the measured value of the normalized velocity variance is 0.0025 , which is shown as a circle on the $\alpha=0$ axis. The normalized variance resulting from the polydispersity of the suspension was calculated to be 0.001 , which is even smaller than the variance produced by the bubble trajectory oscillations.

To further investigate the rapid change in the bubble velocity and velocity variance as the concentration tends to zero, a series of experiments was performed in a very dilute suspension. Bubble concentrations smaller than $\alpha=0.001$ cannot be measured accurately. For such concentrations the increase in the height of the column resulting from the introduction of the bubbles is less than $1 \mathrm{~mm}$, the minimum length that can be measured accurately. The impedance probe becomes inadequate for such dilute systems since bubbles collide infrequently with the probe and the recording time required to obtain statistically significant results becomes impractical. Instead the bubble motion was determined from video images and the gas volume fraction regime will be henceforth referred to as 'very dilute'.

In very dilute suspensions, the bubbles were observed to undergo oscillating tra- 


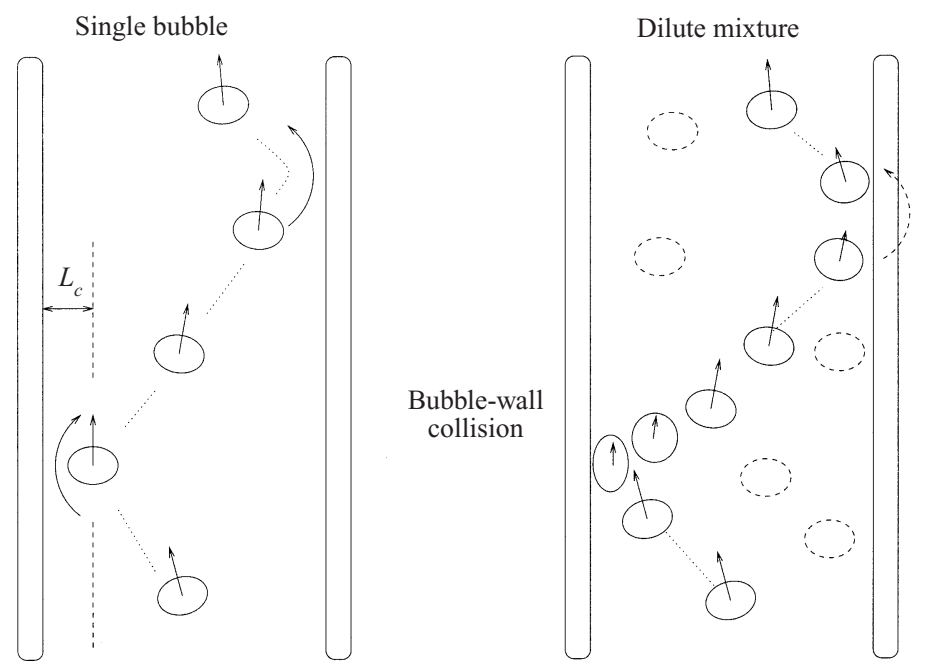

FIGURE 11. Schematic diagram of the motion of a single bubble in the channel and of a bubble in a very dilute suspension. Dashed lines indicate the presence of other bubbles in the suspension that increase the velocity fluctuations of the test bubble. Schematic is not to scale.

jectories. However, in contrast with the single bubble measurements, direct collisions against the containing walls were observed in the very dilute suspension. During a bubble-wall collision the bubble velocity decreases significantly, in accordance with the observation of Tsao \& Koch (1997). When colliding, the bubbles slowed down by as much as $75 \%$ of their original velocity and the shape of the bubble was altered significantly. The velocity decrease as a result of the collision was calculated for a number of bubble collisions. The ratio of the velocities after and before the collision, was 0.25 for the vertical component and 0.74 for the horizontal component.

As a result of the collisions with the containing walls in the very dilute suspension, the mean bubble velocity decreased to $0.288 \mathrm{~m} \mathrm{~s}^{-1}$ while the normalized bubble velocity variance increased to 0.012 (denoted by the diamond symbols in figures 9 and 10 respectively). These values are intermediate between the values obtained from single bubble experiments and the suspension experiments with $\alpha>0.01$. In a narrow region, at very low gas concentration, the bubble velocity and variance change rapidly resulting from the bubble-wall collisions. Clearly the small increase of gas volume fraction produced an increase in the bubble-bubble interactions which resulted in large enough horizontal velocity fluctuations to cause bubbles to collide with the containing walls. The collisions with the walls are, therefore, responsible for the sudden decrease of the mean bubble velocity at very low concentrations. Figure 11 depicts the observed behaviour schematically.

To corroborate the experimentally measured velocities a comparison can be performed between the superficial gas velocity, $u_{o}=Q / A_{o}$, measured with the gas flow meter and the product $\left(u_{b} \alpha\right)$, of the holdup with the bubble velocity measured by the impedance probe. $Q$ is the volumetric gas flow rate and $A_{o}$ is the cross-sectional area of the channel. The correlation between the two measures is found to be very good, with no more than 5\% discrepancy for all concentrations. At low concentration the gas flow measurements are slightly higher than the values measured directly with the impedance probe but for more concentrated mixtures the measured bubble velocity is always larger than that calculated from the flow rate. 


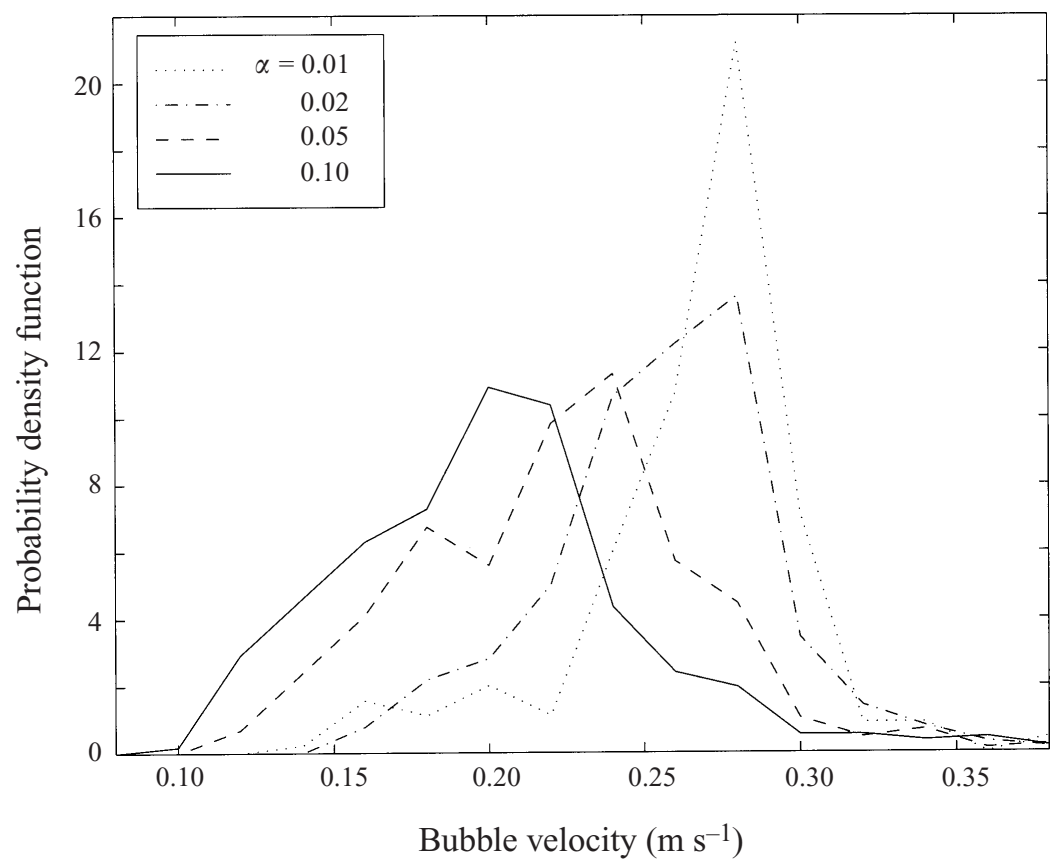

FIGURE 12. Normalized bubble velocity probability density function for four typical gas volume fractions. The width of the bins is $0.02 \mathrm{~m} \mathrm{~s}^{-1}$.

\subsubsection{Probability density functions of bubble velocity}

Probability density functions (PDFs) for the bubble velocity can be constructed from the individual measurements of bubble velocity. Figure 12 shows the PDFs for four different mean gas volume fractions. All the PDFs are normalized such that the area under the curve equals one. For a dilute bubble suspension, the velocity distribution is tall and narrow indicating that the velocity variance is small. As the mean bubble concentration increases, the velocity distribution becomes wider. The maximum of the PDF is observed to shift to lower velocities as the concentration increases in accordance with the mean bubble velocity measurements. Clearly, the shape does not appear to be truncated on either the high or low velocity sides, which shows that the algorithm that discriminates erroneous signals is not eliminating real velocity traces and, therefore, does not affect the results.

\subsubsection{Comparison with theory}

Spelt \& Sangani (1998) derived the drag on a homogeneous suspension of spherical bubbles rising through a fluid. Their result can be written in the form

$$
u_{b}=\frac{u_{\infty}(1-\alpha)}{1+\frac{3}{20} \alpha A}
$$

where $A=u_{b}^{2} / T_{b}$ is the ratio of the mean bubble velocity squared to the bubble velocity variance. Figure 9 shows the prediction from equation (3.5) for two constant values of the parameter $A$ and using a function $A(\alpha)$ obtained from the fit of the velocity variance measurements (from figure 10 ). The predictions are calculated using a value of $u_{\infty}=0.32 \mathrm{~m} \mathrm{~s}^{-1}$, corresponding to the single bubble moving in a large channel. Despite the shift caused by the chosen value of $u_{\infty}$, the comparison is very 
good. The slope of the experimental curve is similar to that predicted by Spelt \& Sangani. The primary difference between the experiment and the theoretical prediction is the rapid decrease in velocity with bubble concentration in the very dilute regime. As discussed above, we attribute this decrease to bubble-wall interactions which are not included in the theory. It should also be noted that the values of $A$ measured in the experiments fall within the regime for which Spelt \& Sangani predict that the homogeneous suspension is unstable to cluster formation so that the applicability of (3.5) is uncertain.

The comparison of the theoretical and experimental results for the dimensional mean bubble velocity shown in figure 9 does not take into account changes in the equivalent diameter and aspect ratio of the bubbles observed in the experiments. To factor out the changes in mean velocity that may arise due to these changes, the mean bubble velocity can be scaled with the terminal velocity $u_{\text {Moore }}$ predicted by Moore (1965) for an isolated bubble with the measured equivalent diameter $d_{e q}$ and aspect ratio $\chi$, i.e.

$$
u_{\text {Moore }}=\frac{d_{e q}^{2} \rho_{f} g}{36 \mu} \frac{1}{G(\chi)}
$$

where $G(\chi)$ is a function of the bubble aspect ratio, $\chi$, given by

$$
G(\chi)=\frac{1}{3} \chi^{4 / 3}\left(\chi^{2}-1\right)^{3 / 2} \frac{\left(\chi^{2}-1\right)^{1 / 2}-\left(2-\chi^{2}\right) \sec ^{-1} \chi}{\left(\chi^{2} \sec ^{-1} \chi-\left(\chi^{2}-1\right)^{1 / 2}\right)^{2}} .
$$

Using equation (3.2) and a fit of the experimental measurements of the bubble aspect ratio, the terminal velocity for the oblate bubbles can be inferred as a function of the gas volume fraction. The normalized bubble velocity, $u_{b} / u_{\text {Moore }}$ is shown in figure 13 and is compared with expression (3.5) normalized by $u_{\infty}$,

$$
\frac{u_{b}}{u_{\infty}}=\frac{(1-\alpha)}{1+\frac{3}{20} \alpha A} \text {. }
$$

Three different values of the parameter $A$ were chosen: $A=10, A=20$ and a fit from the experiments, $A(\alpha)$.

As in the comparison of dimensional mean velocity given in figure 9 , the theory in figure 13 tends to overpredict the bubble velocity. The measured bubble velocity at zero bubble volume fraction is $5.5 \%$ lower than that calculated using Moore's expression. This difference may result from wall effects and possibly from small traces of surface-active contaminants in our system. As the bubble concentration increases, the bubble diameter increases slightly and the bubble shape becomes nearly spherical. This leads to a significant increase in the terminal velocity $u_{\text {Moore }}$. The experimental measurements suggest that the trend toward more spherical bubbles with increasing bubble concentration does not decrease the drag force as much as might be expected. It should be noted that while the bubbles in the more concentrated suspensions are nearly spherical on average, they are constantly undergoing bubble-bubble collisions that excite small-to-moderate amplitude bubble shape oscillations. The dissipation of energy associated with these shape oscillations is one possible explanation for the higher drag observed in the experiments as compared with the theory for perfectly spherical bubbles. This possibility could be explored using numerical simulations for deformable bubbles with potential flow hydrodynamic interactions and direct collisions between the bubbles.

It is interesting to compare the change in the normalized mean velocity of the bubbles in our experiment with that predicted for other types of suspensions. At 


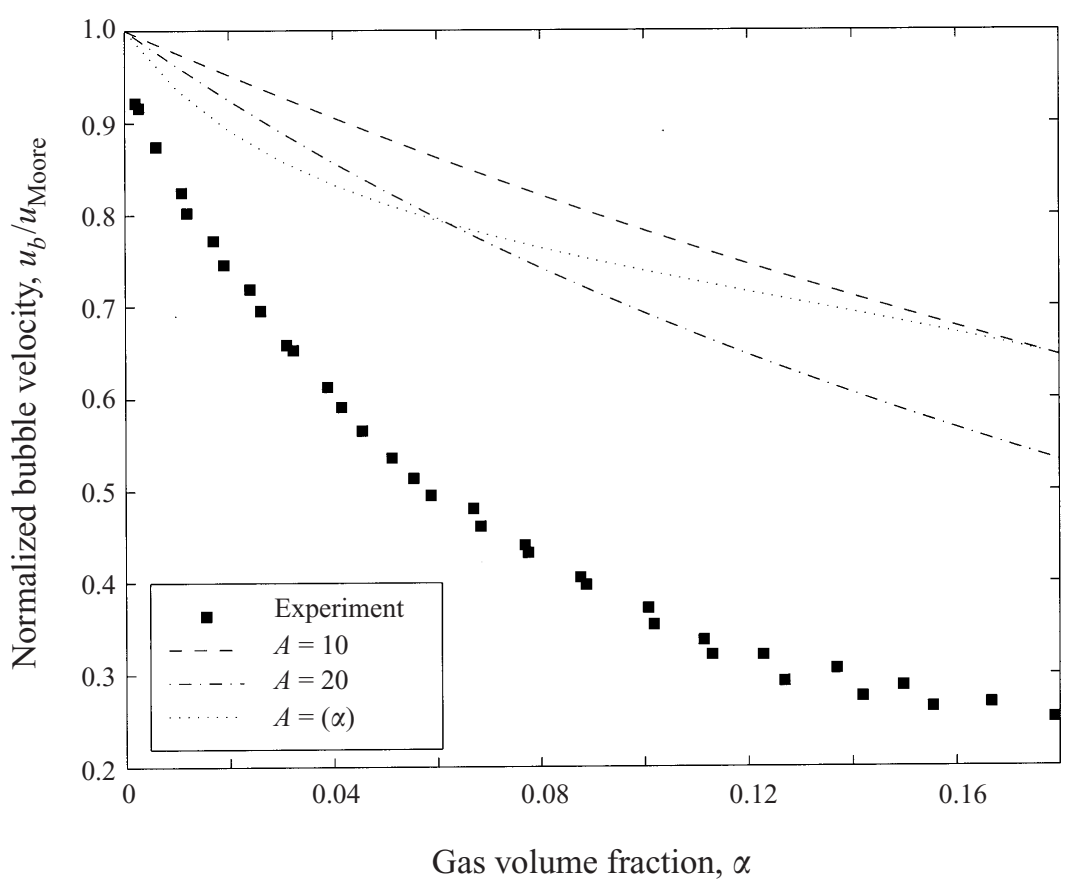

FIGURE 13. Normalized bubble velocity, $u_{b} / u_{\text {Moore }}$ as function of gas volume fraction.

$\alpha=0.18$, the experimental measurements indicate a normalized bubble velocity of 0.25 compared with the prediction of 0.65 for spherical bubbles with potential flow interactions, cf. figure 13. In fact the measured normalized bubble velocity is comparable with the hindered settling velocity for a solid sphere in a low Reynolds number flow (Durlofsky \& Brady 1988; Ladd 1990). In addition to the role of bubble deformation, another possible scenario that could lead to the large decrease in normalized bubble mean velocity observed in the experiments would be an increase in deviations from potential flow as the suspension becomes more concentrated. It is known that the potential flow approximation provides an accurate description of the rise of a single bubble (Moore 1965; Duineveld 1995), but much less is known about its accuracy in suspension flows. It is possible that the more complex flow patterns in a suspension and the cumulative effect of vorticity generation at many bubble surfaces lead to deviations from potential flow. It should also be noted that the case of bubbles rising steadily through a fluid would lead to larger deviations from potential flow than would occur in accelerating flows or flows with a larger ratio of the fluctuating to the mean bubble velocity relative to the liquid. Noting that the drag on a single solid sphere at the lowest Reynolds number encountered in our experiments $(R e=280)$ is nearly four times larger than the drag on a spherical bubble in potential flow (Clift, Grace \& Weber 1978), it can be seen that the deviations from potential flow would not need to be extreme to lead to the decrease in bubble velocity observed experimentally. As noted earlier the exponent in a Richardson-Zaki type correlation for the bubble velocity (3.4) is larger $(n=2.796)$ in the present experiments than Richardson \& Zaki's (1954) correlation for solid particles $(n=2.5)$ or Ishii et al.'s correlation for drops and bubbles $(n=2.0)$. Ishii et al. do not specify whether their correlation corresponds to drops or bubbles and do not specify the Weber number. It is not surprising that the high Reynolds number, low Weber number bubbles 
exhibit stronger hindering than other types of suspensions. Bubbles in this special type of inertial suspension experience a very small $O\left(12 \pi \mu a u_{\infty}\right)$ drag compared with the $O\left(\rho a^{2} u_{\infty}^{2}\right)$ inertial drag characteristic of other high Reynolds number particles. Thus, the high Reynolds number, low Weber number bubble velocities are more susceptible to decrease due to any new dissipation mechanisms that may arise due to bubble-bubble interactions.

Van Wijngaarden (1999) speculated that close interaction between moderate Weber number bubbles may lead to vortex shedding when a non-interacting bubble would produce very little vorticity. This vortex shedding may enhance the drag in a concentrated suspension considerably.

It should be noted that the video images used to determine the bubble diameter and aspect ratio in the more concentrated suspensions only probe the bubbles near the channel walls. Thus, it could be argued that the bubbles in the middle of the channel may have a different size or aspect ratio than that measured by the image analysis. However, we do not believe that this effect is significant because we observed no statistically significant change in the mean bubble velocity and volume fraction measured by the impedance probe with position across channel gap.

\subsection{Bubble-probe collision rate}

Another quantity that can be used to characterize a bubble suspension is the bubble collision rate. The collision rate quantifies the number of bubble events detected by the probes per unit time. Unlike the measurement of bubble volume fraction by an impedance probe with a small measuring volume, the collision rate does not require a consideration of the residence time of the bubbles in the measuring volume. In this sense, collision rate is a simpler quantity to measure. The collision rate can easily be predicted from kinetic theories and is related to the volume fraction, mean velocity and velocity variance.

With the present experimental arrangement, the bubble collision rate can be detected using either the impedance or the hot-wire probe. The measurements can then be compared with each other by normalizing the collision rate by the cross-sectional area for bubble collision with the sensor's sensitive area. The collision detection area is determined by the single bubble experiments in which bubbles were released at different horizontal positions relative to the probe. The range of horizontal positions for which the probe signal rose above a specified threshold level was then determined. The sensitive area for the hot-wire probe was measured to be $1.803 \mathrm{~mm}^{2}$ for a velocity slope threshold level of $-300 \mathrm{~m} \mathrm{~s}^{-2}$, and the sensitive area for the impedance probe was measured as a function of the bubble diameter as denoted by

$$
A_{\text {coll }}=0.611 d_{\text {long }}^{2}-1.588 d_{\text {long }}+1.747
$$

where the units of $d_{\text {long }}$ and the collision detection area are $\mathrm{mm}$ and $\mathrm{mm}^{2}$, respectively. This expression was obtained for a threshold level of $0.2 \mathrm{~V}$.

Figure 14 shows the number of collisions per unit time per probe area as a function of the mean gas volume fraction. The collision rate rises with increasing bubble number density as expected. The slope of the collision rate curve decreases as the gas volume fraction increases. This results from the decrease in the mean bubble velocity with gas volume fraction. The measurements from the two probes agree very well for small bubble concentration but begin to differ for concentrations larger than 0.05 . This may result from differences in the nature of bubble interactions with the two probes owing to differences in the probe geometries. The sensitive hot-wire probe is supported by two posts (see figure 3) which may shield the probe from 


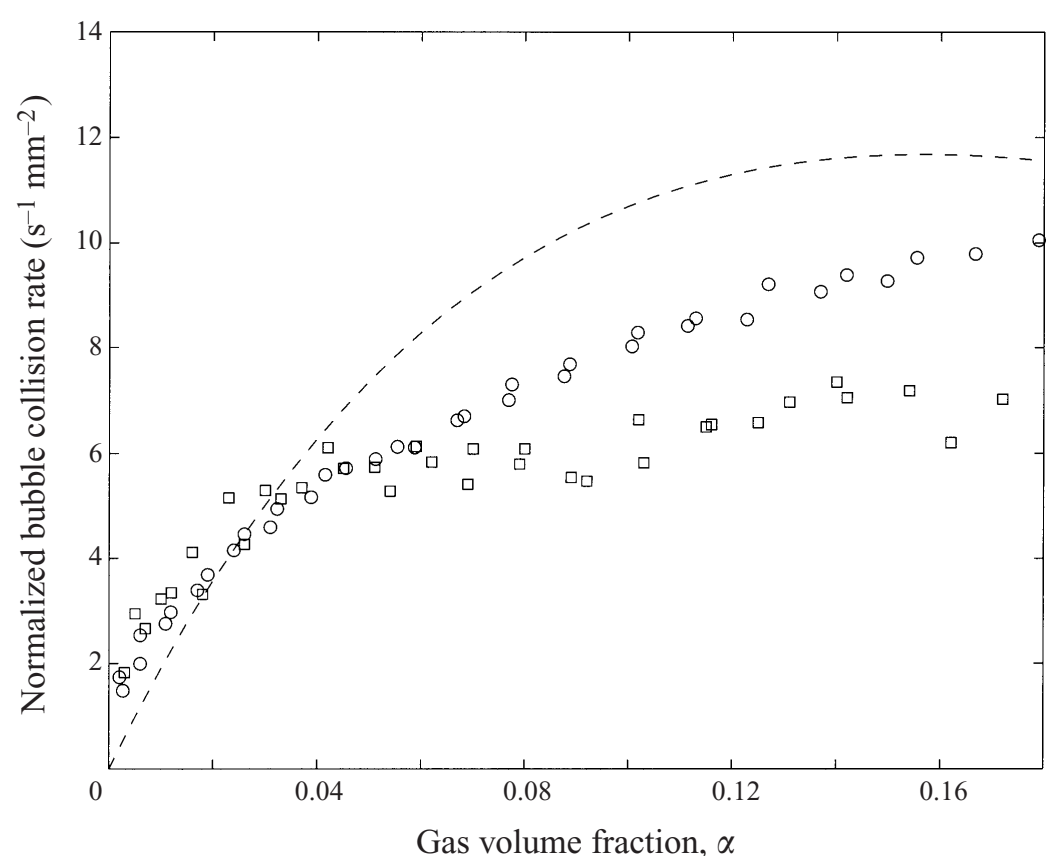

FIGURE 14. Number of collisions per unit time per probe area, or normalized collision rate, as a function of mean gas volume fraction. The circles represent the measurements obtained with the impedance probe normalized by the collision detection area from equation (3.7). The squares show the measurements obtained with the hot film probe (aligned horizontally) normalized by a constant collision detection area of $2.513 \mathrm{~mm}^{2}$. The dashed line is the prediction from equation (3.9).

collisions by bubbles with a significant horizontal velocity component. In contrast the impedance probe (figure 5) restricts bubble collisions from a significantly smaller part of angular space. The increasing velocity variance of the bubbles in more concentrated suspensions (illustrated in figure 14) may lead to more collisions from bubbles with larger horizontal velocity components that can be more easily detected by the impedance probe.

Assuming that the bubbles all have the same velocity, the collision rate can be expressed in terms of the bubble velocity, volume fraction and equivalent diameter as

$$
C_{r}=\frac{6}{\pi} \frac{\alpha u_{b}}{d_{b}^{3}} .
$$

Using equations (3.2) and (3.4), $C_{r}$ can be calculated and compared with the direct experimental measurements.

Video image processing was used to evaluate the magnitude of the vertical and horizontal bubble velocity variances. This technique, however, could only be used when the bubble suspension was dilute. The ratio of vertical to horizontal bubble velocity variance was measured to be 2.01 for a volume fraction of 0.005 , and 1.75 for a volume fraction of 0.014 . Clearly, the component of the bubble velocity variance in the horizontal direction is smaller and has a small contribution to the bubble-probe collision rate. Hence, a more sophisticated estimate of the collision rate that takes account only of the fluctuations in the vertical bubble velocity can be obtained by assuming that the velocity takes on a Maxwellian distribution and has components 
only in the vertical direction:

$$
f_{M}\left(c_{z}\right)=\frac{1}{\left(2 \pi T_{b}\right)^{1 / 2}} \exp \left[\frac{-\left(c_{z}-u_{b}\right)^{2}}{2 T_{b}}\right]
$$

where $c_{z}$ is the $z$-component of the bubble velocity. The collision rate can then be obtained by integrating

yielding

$$
C_{r}=\int n f_{M}\left(c_{z}\right)\left|c_{z}\right| \mathrm{d} c_{z}
$$

$$
C_{r}=\frac{6 \alpha}{\pi d_{b}^{3}}\left[\sqrt{\frac{2 T_{b}}{\pi}} \exp \left(-u_{b}^{2} / 2 T_{b}\right)+u_{b}\left(\operatorname{erf}\left(u_{b} / \sqrt{2 T_{b}}\right)\right)\right] .
$$

This expression can be evaluated using the measurements of the bubble volume fraction and the mean and variance of the bubble velocity and it is represented by the dashed line in figure 14 . The contribution of the velocity variance to the collision rate obtained from equation (3.9) was found to be on the order of $0.01 \%$.

The measurements from both probes deviate from the prediction for gas volume fractions greater than 0.05 . The differences between the measured value of the collision rate and the predictions may be an indication of the shielding produced by the probe supports.

\subsection{Liquid velocity variance}

The liquid velocity variance was determined from the hot-wire signal at various values of the mean gas volume fraction. The sensor was positioned in the middle of the channel and measurements were taken with the probe axis oriented both horizontally and vertically so as to obtain information concerning the vertical $\left(T_{f v}\right)$ and horizontal $\left(T_{f h}\right)$ velocity variances. As discussed in $\S 2.2$, it is assumed that the probe signal responds primarily to velocity components perpendicular to the probe axis. Thus, the signal obtained when the probe is oriented vertically is a measure of $2 T_{f h}$ and when the probe axis is horizontal $T_{f h}+T_{f v}$ is measured.

Figure 15 shows $T_{f v}$ and $T_{f h}$ normalized by the square of the mean bubble velocity $u_{b}^{2}$ as a function of the mean gas volume fraction. As expected, the liquid velocity variance increases with gas volume fraction, since there are more bubbles to create fluid velocity disturbances. The vertical velocity variance is larger than the horizontal velocity variance for all gas volume fractions as is usually the case in sedimenting suspensions.

A prediction of the liquid velocity variance in a dilute suspension of homogeneously distributed bubbles can be obtained by calculating the potential-flow fluid velocity variance produced by a single spherical bubble translating through an unbounded fluid. The potential function for a sphere of radius, $a=d_{b} / 2$, moving at a velocity $u_{b}$ is given by

$$
\phi=\frac{1}{2} \frac{a^{3}}{r^{3}} \boldsymbol{x} \cdot \boldsymbol{u}_{b} .
$$

The fluid velocities in the vertical and horizontal directions are calculated as

$$
u_{x}=\frac{\partial \phi}{\partial x}, \quad u_{y}=\frac{\partial \phi}{\partial y}
$$

where $x, y, z$ are Cartesian coordinates with the $x$-axis parallel to gravity. To obtain the fluid velocity variance, the square of the velocity disturbance is integrated over all 


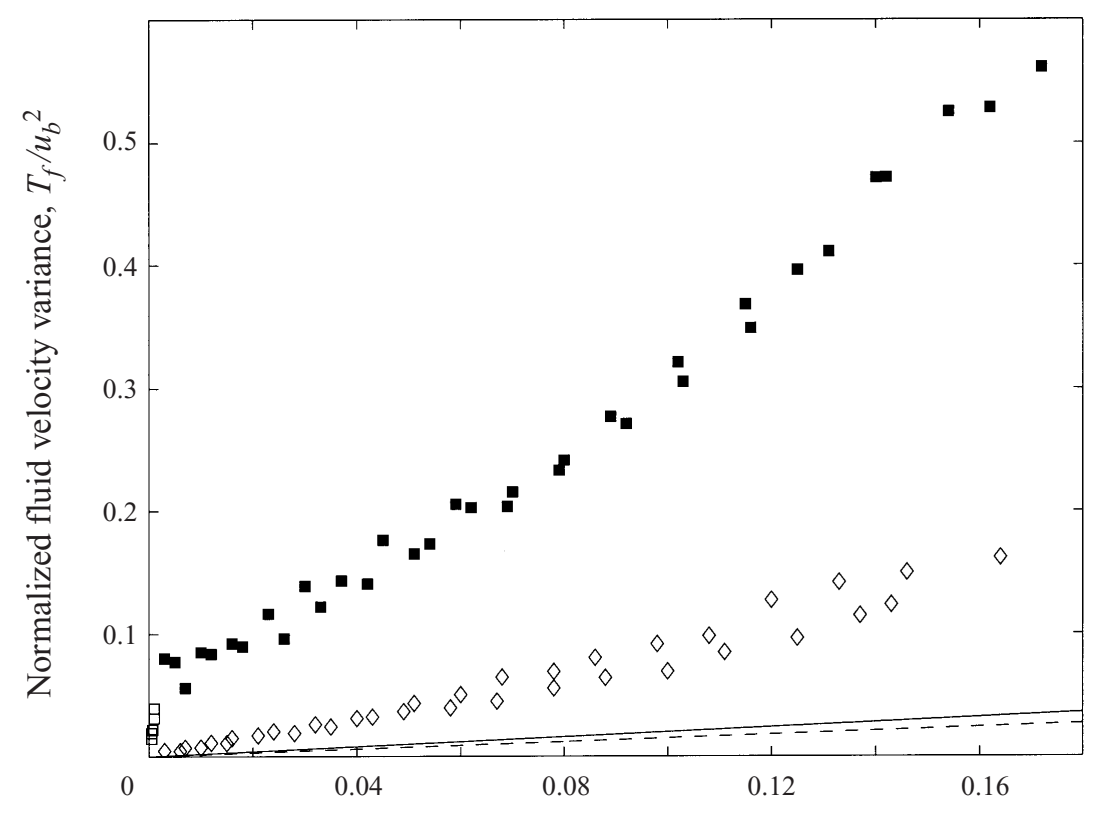

Gas volume fraction, $\alpha$

FIGURE 15. Normalized fluid velocity variance as a function of gas volume fraction. The solid squares show the vertical velocity variance, $T_{f v}$, and the diamonds depict the horizontal velocity variance, $T_{f h}$. The open squares represent the vertical velocity variance measured in the 'very dilute' limit. The solid line shows the prediction of the vertical fluid velocity variance from equation (3.11), and the dashed line shows the horizontal fluid velocity variance from equation (3.12).

space and multiplied by the bubble number density,

yielding

$$
\begin{gathered}
\left\langle u_{x}^{2}\right\rangle=n \int_{V} u_{x}^{2} \mathrm{~d} V, \\
\left\langle u_{y}^{2}\right\rangle=n \int_{V} u_{y}^{2} \mathrm{~d} V, \\
\left\langle u_{x}^{2}\right\rangle=\frac{1}{5} \alpha u_{b}^{2}, \\
\left\langle u_{y}^{2}\right\rangle=\frac{3}{20} \alpha u_{b}^{2} .
\end{gathered}
$$

The predictions from equations (3.11) and (3.12) are shown in figure 15 by the solid and dashed lines, respectively. Both the theoretical predictions for a dilute, homogeneous suspension and the experimental measurements indicate that the vertical velocity variance is substantially larger than the horizontal. However, this simple theory under-estimates the liquid velocity variance at all bubble concentrations. At the higher bubble concentrations, this discrepancy could result from the details of the bubble-bubble interactions that are neglected in the model. However, the large velocity variance in a dilute suspension suggests that the suspension may not be homogeneous. Clusters of bubbles in a dilute suspension would be expected to produce a larger liquid velocity disturbance than homogeneously distributed bubbles. This observation can be inferred from the simulation results of Sangani \& Didwania (1993b), where the added mass coefficient was calculated and it was observed to increase as the bubble clusters formed. 
The non-spherical nature of the bubbles also causes the liquid velocity variance to be larger than that predicted for spherical bubbles. We can get an estimation of magnitude of this effect by comparing the added mass coefficient (which is the kinetic energy of the liquid) for spherical bubbles to that for oblate spheroids resembling the bubbles in the experiments. For example, the added mass coefficient for an oblate spheroid of aspect ratio 1.5 is $4 / 5$, while that for a sphere is $1 / 2$. Hence, in this case, the nonsphericity of bubbles would be expected to increase the velocity variance by $60 \%$ and thereby explain a small portion of the discrepancy between the theory and experiment.

Note that, as in the case of the measurement of the bubble velocity variance, the vertical liquid velocity variance appears to approach a finite value as the concentration of bubbles approaches zero. A series of measurements was performed for the "very dilute' limit in which the concentration cannot be measured accurately (shown by the open squares in figure 15). It was found that the fluid velocity variance rapidly increases for that range of concentrations. One would expect the liquid velocity variance to approach zero as $\alpha \rightarrow 0$ in a homogeneous suspension. However, it will be seen in the following section that the bubbles form clusters. The rapid increase of the vertical fluid velocity variance at small concentrations may arise from the instability of the suspension, particularly if clustering is most extreme for the smallest volume fractions.

Sangani \& Didwania (1993b) simulated suspensions of rising bubbles with an initially random spatial distribution. The bubbles formed clusters as they rose, resulting in a substantial increase in the added mass coefficient or liquid velocity variance. The increase in this velocity variance with clustering was greater at smaller volume fractions, which is consistent with our experimental observations.

\subsection{Liquid velocity probability density function}

From the hot-wire measurements, we can construct probability density functions (PDFs) for the velocity signal obtained when the probe axis is oriented horizontally or vertically. Recall that the vertically oriented probe measures the horizontal liquid velocity. The horizontally oriented probe detects the vertical velocity and one component of the horizontal velocity. Since the vertical velocity fluctuations are significantly larger than the horizontal velocity fluctuations (cf. figure 15), the velocity signal from the horizontally oriented probe will be affected primarily by the vertical velocity fluctuations.

Figure 16 shows the normalized PDF of the velocity signal shown in figure 4 for a probe oriented horizontally in a bubble suspension with a volume fraction of 0.05 . The experimentally determined PDF curve has a maximum at an intermediate value of the fluid velocity. The shape of the PDF resembles that of a two-dimensional Maxwellian:

$$
P(\bar{v})=\left(\frac{1}{2 \pi T_{f}}\right) \bar{v} \exp \left(\frac{-\bar{v}^{2}}{2 T_{f}}\right)
$$

where $P(\bar{v})$ is the PDF of the fluid speed, $\bar{v}$, and $T_{f}$ is the variance of the fluid velocity. The two-dimensional Maxwellian is shown in figure 16 along with the experimentally determined PDF. The experimental PDF has a longer tail at high velocities than the Maxwellian. In a dilute suspension, one would expect a small number of events in which the bubble passes close to the probe producing a large velocity and these close passes may account for the tail of the PDF. It is also possible that the direct collisions of the bubble with the probe produce some of the high velocity signals.

Figure 17 shows the normalized PDFs for the velocity signal when the probe is oriented horizontally and vertically for four values of the gas volume fraction. Recall that the horizontally oriented probe primarily measures the vertical velocity 


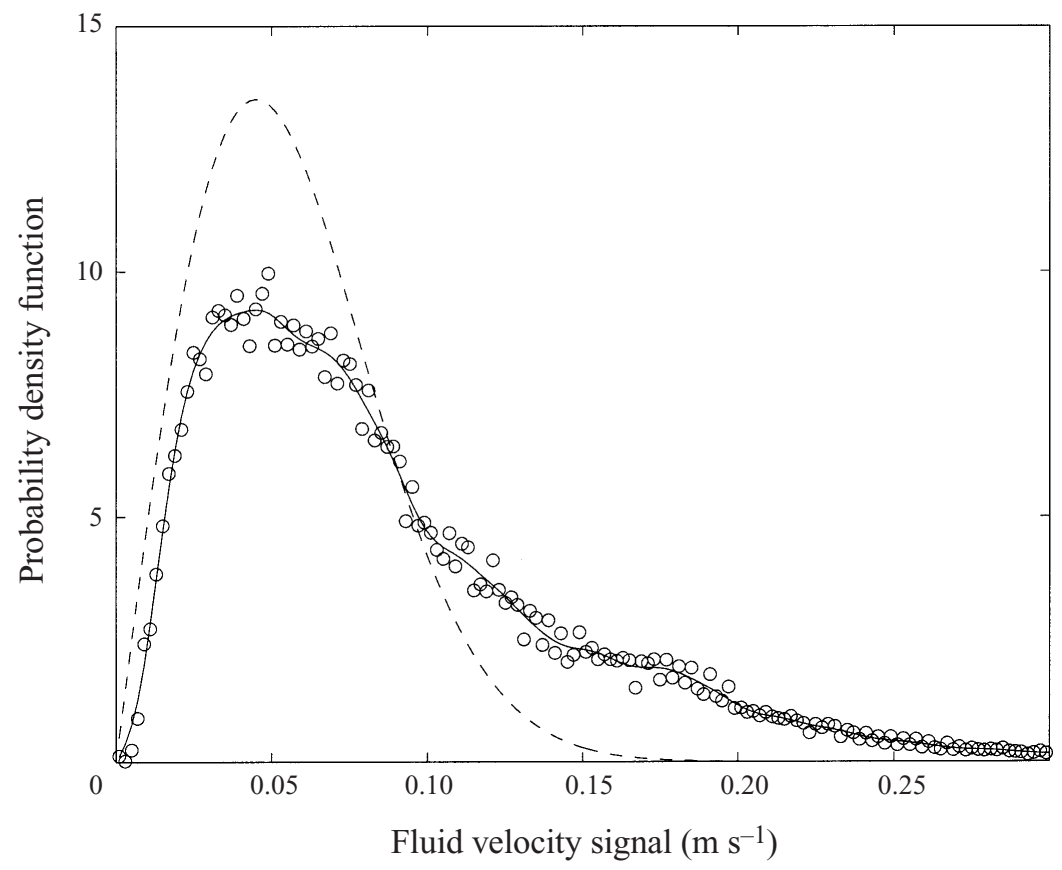

FIGURE 16. Probability density function of the fluid velocity signal for a mean gas volume fraction of 0.05 . The circles represent the experimental measurement for the case when the axis of the hot wire is horizontal; the solid line is a fit to the experimental results. The dashed line is the PDF for the speed of a two-dimensional isotropic Maxwellian defined in (3.13) with the same value of the most probable speed as in the experimental PDF.

fluctuations while the vertical probe detects horizontal velocity fluctuations. The PDFs have a clear maximum that shifts to higher velocities as the bubble concentration increases. As expected, the maximum is at a higher velocity for the probe that detects vertical liquid velocity fluctuations. The PDFs have long tails at high velocities, which may result from the signal produced by direct bubble-probe collisions. In the single bubble tests, it was observed that bubble collisions with the hot wire were characterized by a large change in signal slope that was accompanied by velocity signal spikes. In suspensions with $\alpha \leqslant 0.05$, a second peak in the PDFs at higher velocities is observed in the signal that detects vertical velocity fluctuations. This peak, which occurs for velocities of approximately $0.2 \mathrm{~m} \mathrm{~s}^{-1}$, may be attributed to events where a bubble moving with a velocity close to the mean velocity passes within a bubble diameter of the probe.

\subsection{Power spectrum of the liquid velocity}

To obtain information concerning the temporal correlations of the liquid velocity, we consider the power spectrum of the liquid velocity signal, obtained from the discrete temporal Fourier transform of the signal. We will consider the power spectrum for the fluid velocity signal from the horizontally oriented hot-film probe. This signal is most sensitive to the vertical fluid velocity and is approximately equal to the absolute

FIGURE 17. Probability density functions of the fluid velocity signal for four typical gas volume fractions. (a) Horizontally oriented probe, which is affected primarily be vertical velocity fluctuations. (b) Vertically oriented probe which measures horizontal liquid velocity fluctuations. 


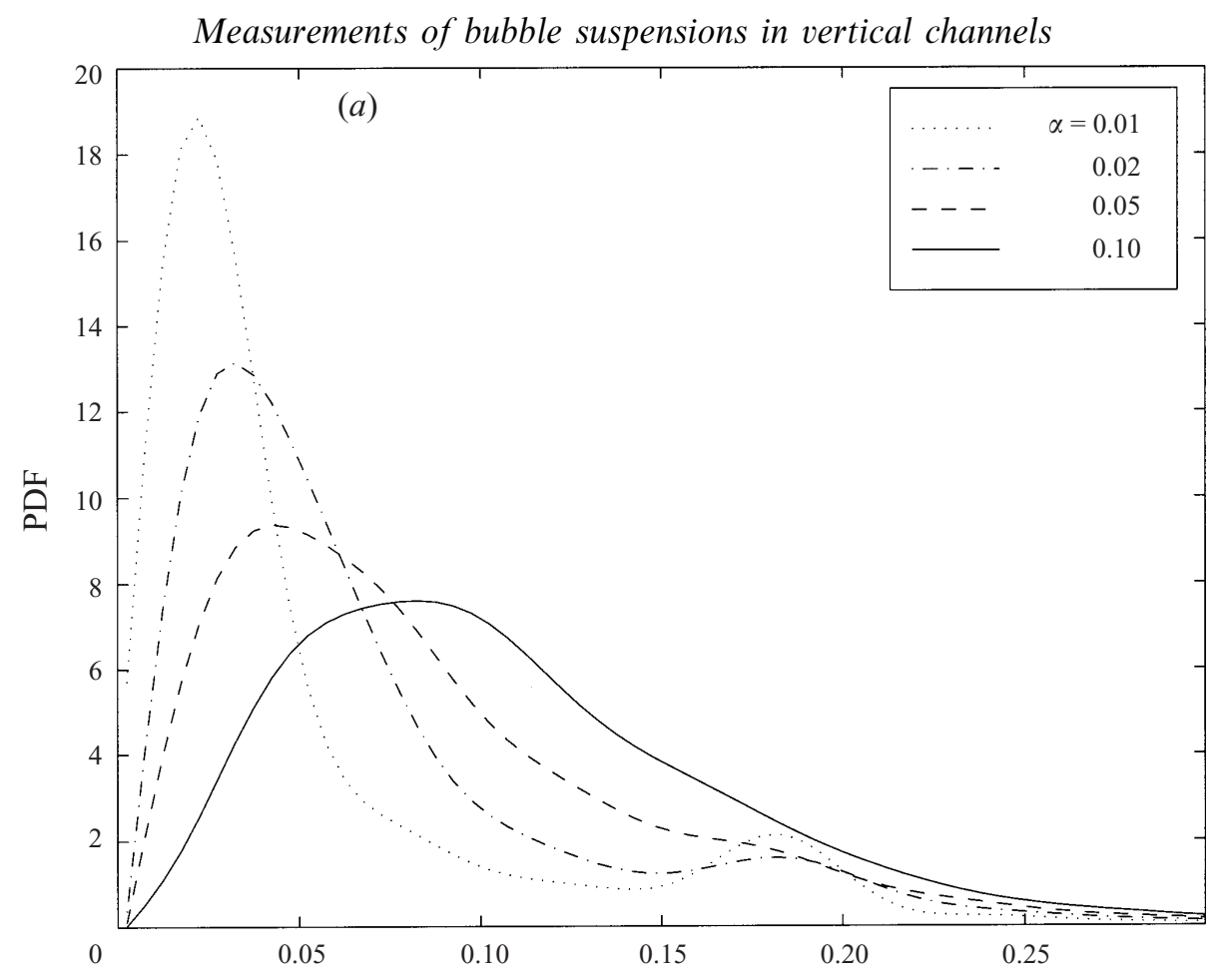

Fluid velocity signal $\left(\mathrm{m} \mathrm{s}^{-1}\right)$

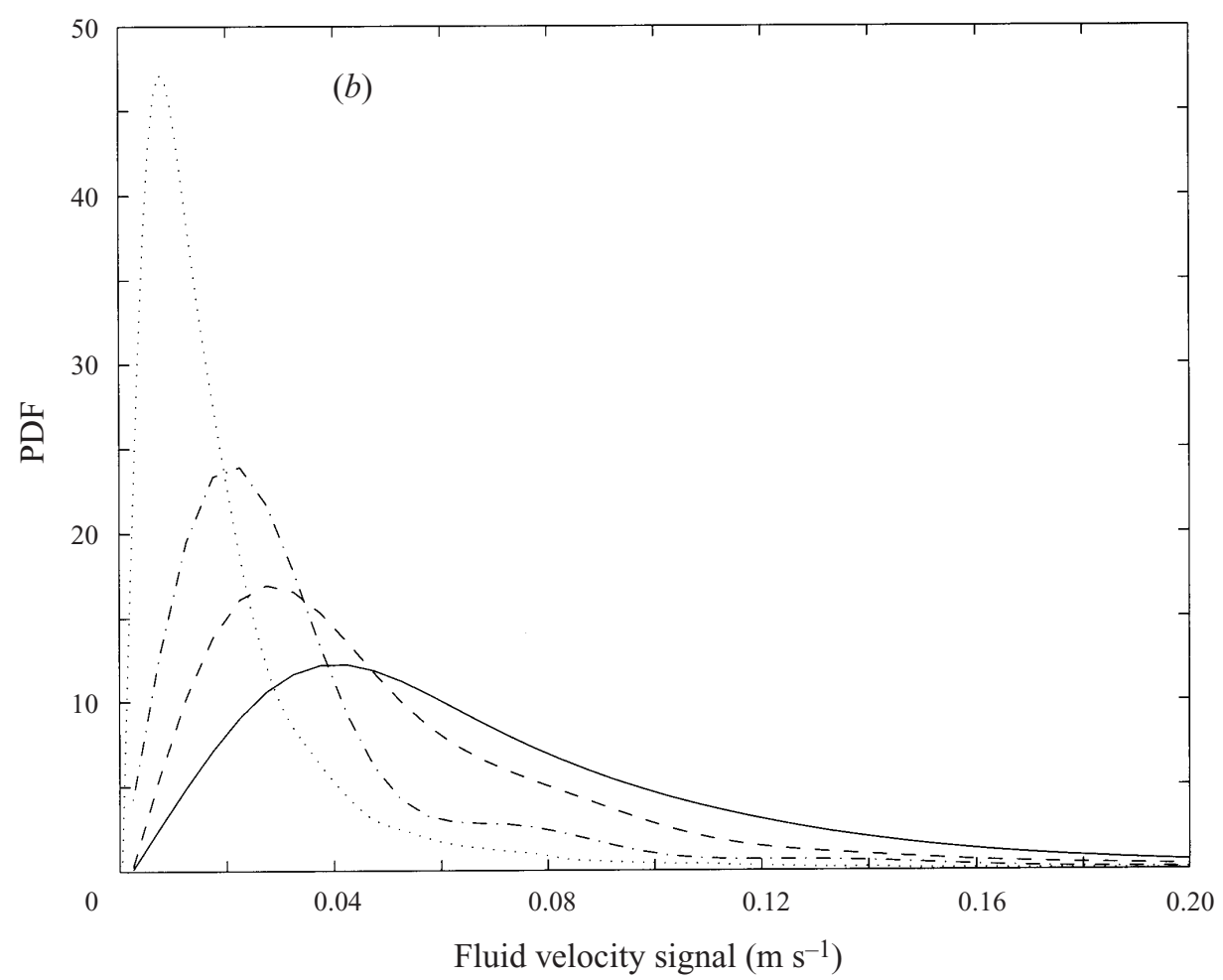

Figure 17. For caption see facing page. 
value of the vertical velocity. The discrete Fourier transform was defined as

$$
\mathscr{V}(\omega)=N^{-1} \sum_{t_{n}=0}^{N-1} v^{\prime}\left(t_{n}\right) \exp \left(-2 \pi \mathrm{i} \omega t_{n}\right)
$$

where $N$ is the number of samples, $t_{n}$ is the time corresponding to the $n$th sample, and $v^{\prime}$ is the deviation of the liquid velocity signal from the mean. The discrete frequency is calculated by $\omega=t_{n} /(\Delta t N)$, where $\Delta t$ is the time between samples.

The power spectrum is defined as

$$
|\mathscr{V}(\omega)|^{2}=\mathscr{V}(\omega) \mathscr{V}^{*}(\omega)
$$

where $\mathscr{V}^{*}(\omega)$ is the complex conjugate of $\mathscr{V}(\omega)$. Figure 18 shows the calculated power spectra for four typical gas volume fractions. The power spectrum of the fluid velocity may be interpreted as a measure of the energy contained in the fluctuations occurring at different frequencies. Because, the hot-film probe is not able to distinguish between upward and downward fluid velocities, the power spectrum in figure 18 is for the absolute value of the vertical fluid velocity. Typically, one may expect the spectrum of the absolute value to be shifted to frequencies that are approximately two times higher than the spectrum for the velocity itself. However, this modest shift will not affect the qualitative interpretations discussed below. The vertical dashed line in the figures indicates the characteristic frequency $\omega_{b}=2 u_{b} / d_{b}$ associated with the passage of an individual bubble near the probe. The dashed-dotted line is the characteristic frequency $\omega_{H}=u_{b} / H$, where $H=2 \mathrm{~cm}$ is the channel gap width. This lower frequency would become relevant if flow structures such as bubble clusters having a size comparable with the channel width passed by the probe at a velocity comparable with the mean bubble velocity.

Clearly, most of the energy in the fluid fluctuations is contained in frequencies lower than the characteristic bubble frequency $\omega_{b}$, and lower than $\omega_{H}$, the frequency associated with the channel gap width. This is observed for all concentrations. These low-frequency fluctuations may result from structures larger than individual bubbles. Therefore, it can be argued that the liquid velocity fluctuations are generated primarily by correlated motions of many bubbles in the suspension rather than by randomly distributed bubbles acting independently. Disturbances of this nature can be attributed to the appearance of clusters of bubbles.

The slope of the power spectrum at frequencies $\omega_{H}<\omega<\omega_{b}$ is more negative at smaller bubble concentrations. In dilute suspensions, the contributions of individual bubbles to the liquid velocity fluctuations is relatively modest and most of the fluctuations come from bubble clusters. This explains the significant fluid velocity variance observed even in quite dilute suspensions in figure 15. As the bubble concentration increases, the liquid velocity fluctuations associated with individual bubbles naturally increase and the tendency toward bubble clustering may decrease somewhat, leading to a smaller slope of the power spectrum. The increased bubble velocity variance and volume fraction will lead to a larger bubble-phase pressure that will tend to decrease the extent of clustering.

For frequencies larger than $\omega_{b}$, the power spectra exhibit an increased slope and the slope in this high-frequency regime is independent of bubble volume fraction. This rapid decrease of high-frequency fluid velocity fluctuations is to be expected because the liquid velocity is produced by structures of the size of bubbles or larger. Note that the frequency response of the hot-wire probe is on the order of $2000 \mathrm{~s}^{-1}$; hence, 
the performance of the probe does not affect the measurement of the fluid velocity fluctuations.

\subsection{Bubble clustering}

Theories and numerical simulations of suspensions of spherical bubbles interacting through potential-flow fluid velocity disturbances indicate that the homogeneous state of the bubble suspension is typically unstable. Sangani \& Didwania (1993b) and Smereka (1993) simulated clouds of bubbles that were initially positioned in a random array within a periodic box. As the simulations progressed in time, the suspension formed horizontal rafts of bubbles that eventually spanned the width of the computational domain. This behaviour is consistent with results for the potentialflow interactions of two bubbles (Biesheuvel \& van Wijngaarden 1982; Kok 1993; Kumaran \& Koch 1994) which predicts that two bubbles aligned within about $55^{\circ}$ to the mean bubble motion repel one another while those aligned closer to the horizontal attract each other.

Spelt \& Sangani (1998) consider the stability of bubble suspensions with a specified bubble velocity variance $T_{b}$ in the absence of viscous dissipation. They considered suspensions to be unstable when the bubble-phase pressure (or trace of the bubble stress tensor) was negative. A rather large value of the bubble velocity variance was needed to stabilize the suspension and the critical value of $T_{b} / u_{b}^{2}$ decreased with bubble concentration. The values of $T_{b} / u_{b}^{2}$ measured in our experiment were generally about a factor of 5 smaller than those necessary to stabilize the suspension.

We have noted above indirect indications of bubble clustering. In particular, it was seen that the liquid velocity variance was much larger than predicted for randomly positioned bubbles and that much of the energy of the liquid velocity spectrum was contained in frequencies that would correspond to the passage of bubble clusters with a size on the order of the channel diameter.

A direct, albeit crude indication of the formation of horizontally oriented bubble clusters can be obtained by analysing images of the bubble suspension taken through the wide face of the bubble channel. The depth of field was large enough that all bubbles across the thickness of the channel were observed except for those shielded by other bubbles. The presence of shielding makes this method of analysis ineffective for $\alpha$ greater than about 0.05 . A software algorithm was used to search and identify bubbles from the digital images. Using the fact that bubbles appear as dark spots in the figures, the images were first converted into binary digital files by choosing a grey threshold level. The pixel values below this threshold were converted into zero denoting the liquid phase; conversely, the pixels with a value greater than the threshold were converted into ones, denoting the bubble phase. Since the centre of the bubble images often had a low gray pixel value, many bubble images were rings. The software searches for these closed convex curves of sizes comparable to the bubble size and changes the inside of the closed curve to a value of 1 . To avoid losing information from the digital processing algorithm, the resulting images were fine tuned manually. Figure 19 shows an example of the result of the image processing. The algorithm was applied to images corresponding to various gas volume fractions. The appearance of horizontal clusters can be quantified in terms of the average pixel value over a horizontal line, $\langle\psi\rangle_{h}$. A pixel row in which a cluster appears will have a value close to 1 while a row with few bubbles will have a value closer to 0 . Figure 20 shows the horizontal average of the pixel value as a function of the vertical position for four typical bubble concentrations. Considerable variation in the mean pixel value with vertical position is observed especially at $\alpha=0.027$ and 0.046 . At higher values 

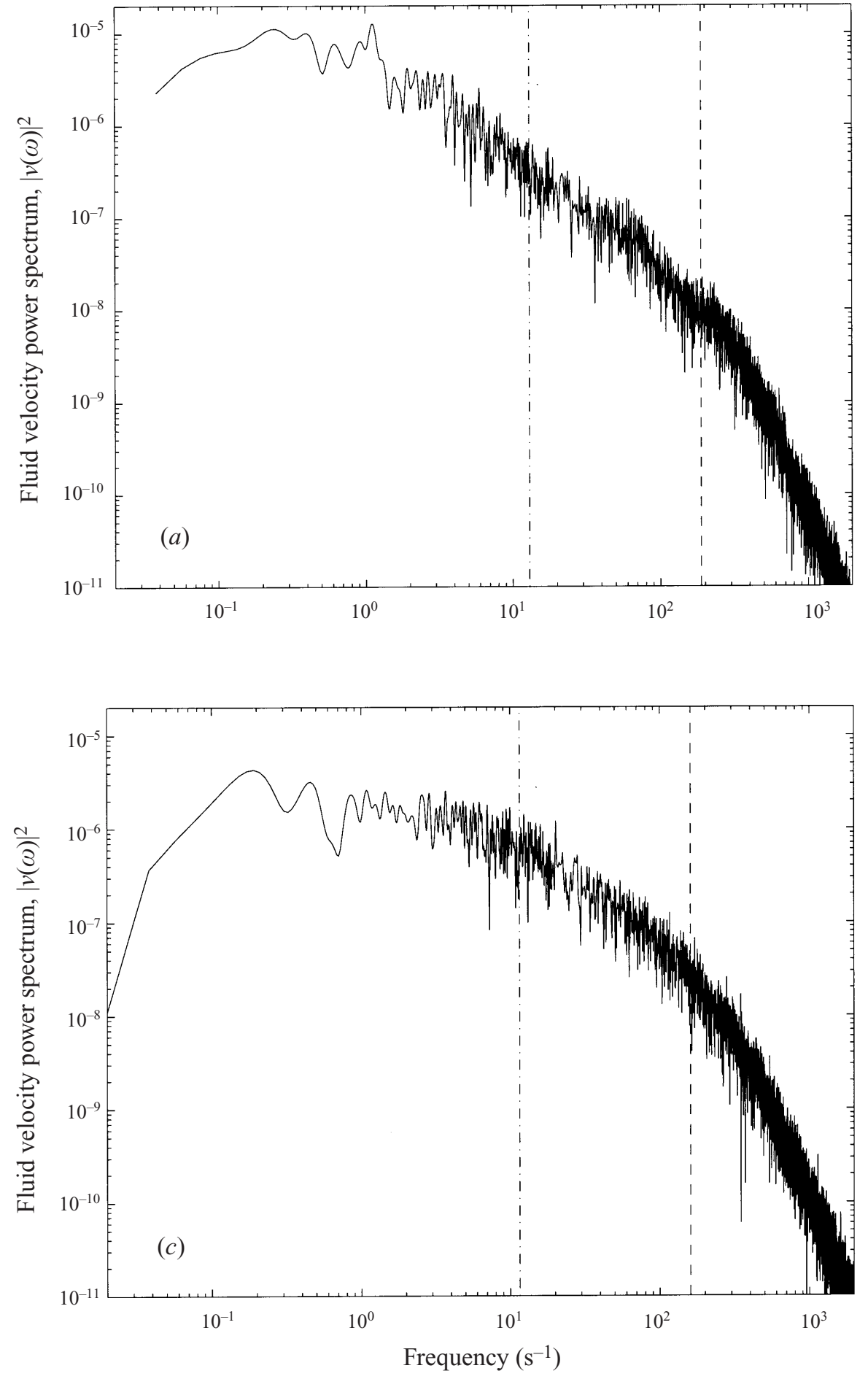

FIGURE 18. For caption see facing page. 
Measurements of bubble suspensions in vertical channels
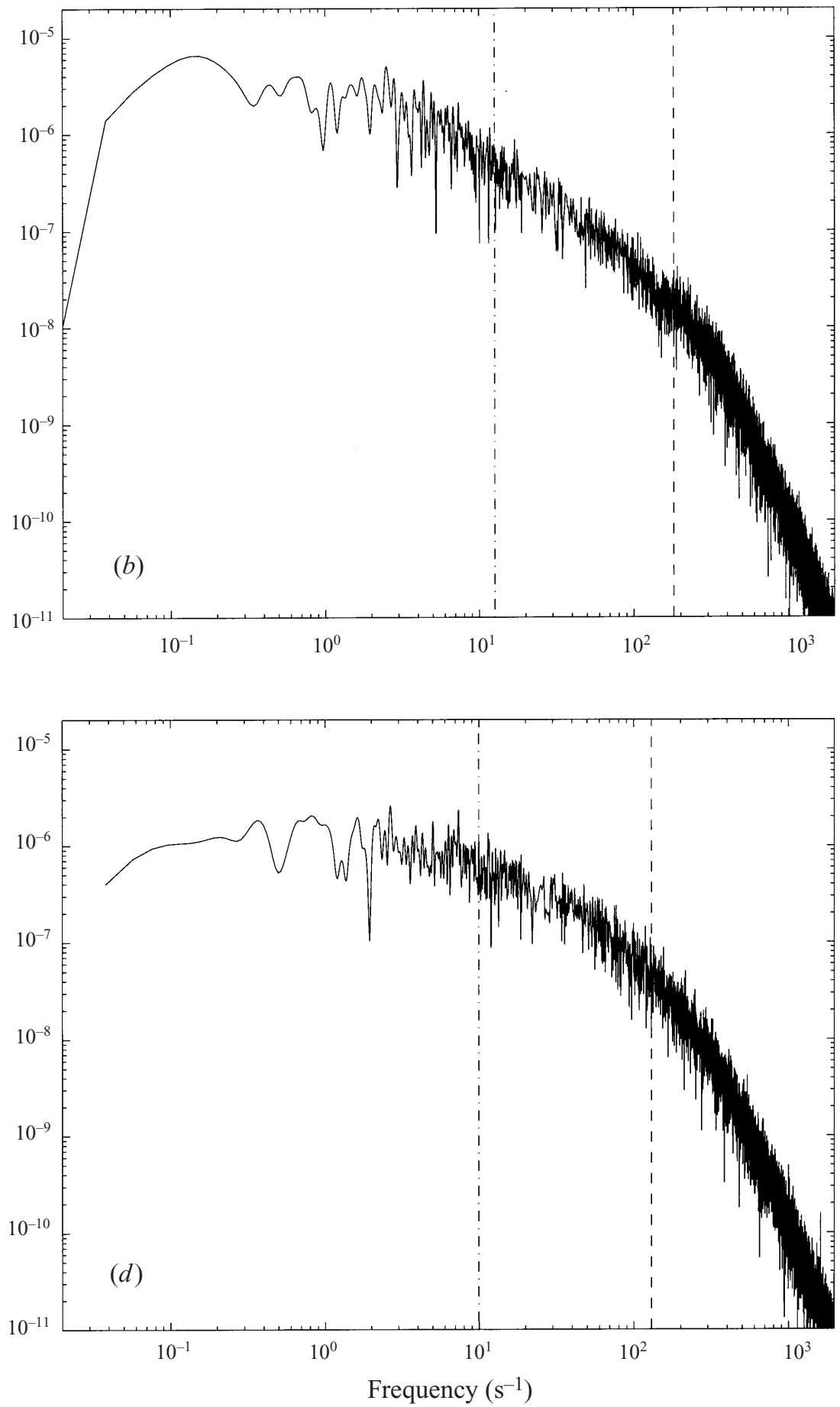

FIGURE 18. Power spectra of the fluid velocity signal for four typical gas volume fractions. The vertical lines denote characteristic frequencies: - -, $2 u_{b} / d_{b} ;-\cdot-, u_{b} / H$ ( $H$ is the gap width). (a) $\alpha=0.01,(b) \alpha=0.02,(c) \alpha=0.05,(d) \alpha=1.10$. 

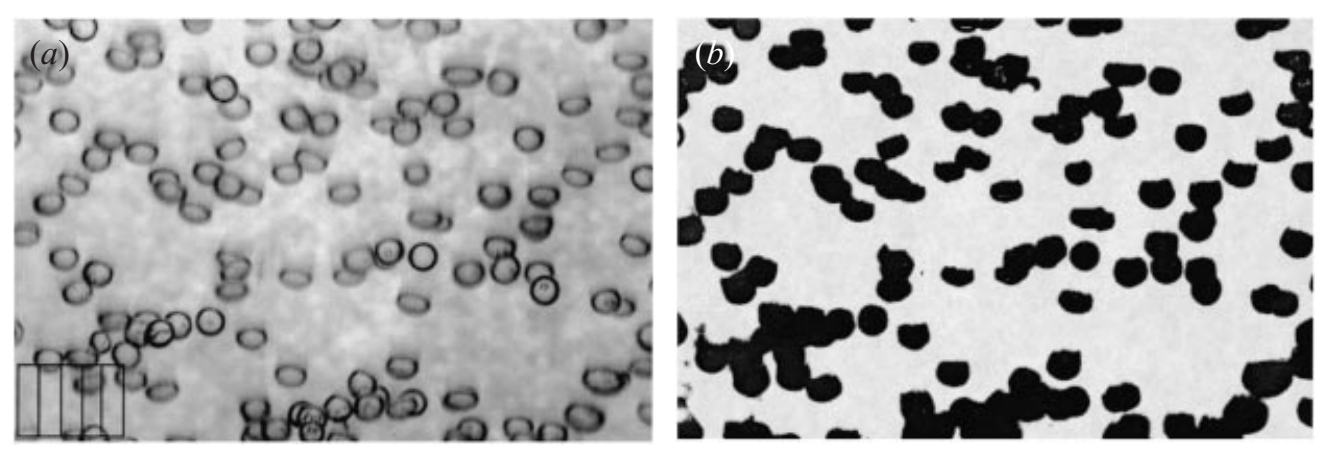

FIGURE 19. Image processing. Photographs are converted into binary images. The spacing in the grid is $1 \mathrm{~mm}$. (a) Original picture, $\alpha=0.02$. (b) Binary image, $\alpha=0.02$.

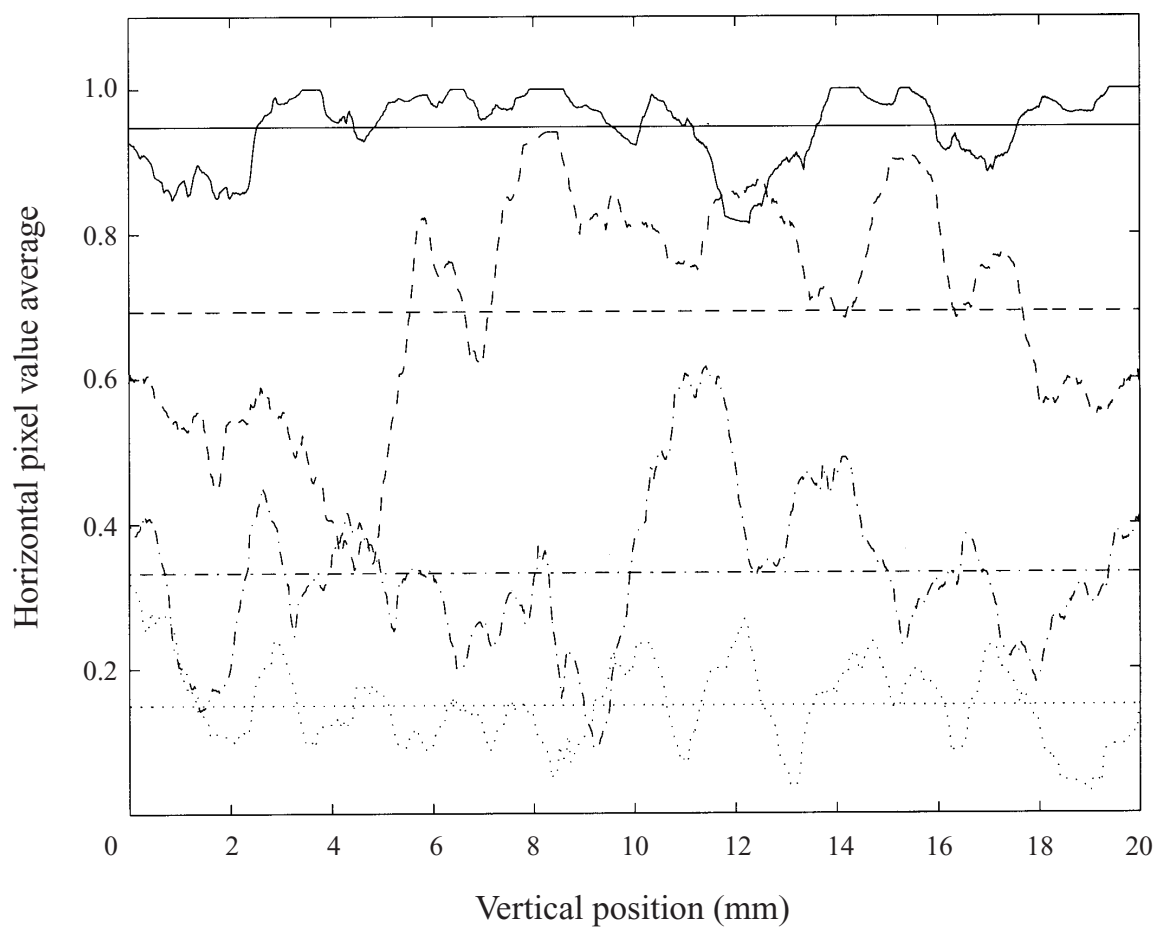

FIGURE 20. Pixel value averaged over a horizontal line, $\bar{\psi}_{h}$, is plotted as a function of vertical position for four different gas volume fractions: $\cdots, \alpha=0.012 ;-\cdot-, \alpha=0.027 ;---, \alpha=0.046$; $-\alpha=0.116$. The horizontal lines show the average pixel value.

of $\alpha$, it is difficult to detect clusters because the image is almost entirely filled with bubbles.

To test for the existence of horizontally oriented clusters, we considered the variance of the horizontal mean pixel value $\Psi_{h}=\left\langle\langle\psi\rangle_{h}^{2}\right\rangle_{v}$ where \langle\rangle$_{h}$ indicates an average over horizontal and \langle\rangle$_{v}$ an average over vertical position. To determine if, in fact, there exists a preferential horizontal structure in the images, the equivalent expression for pixel variance in the vertical direction can be calculated, $\Psi_{v}$, and compared with the one for the horizontal direction, $\Psi_{h}$. The maximum possible size of a cluster in this 


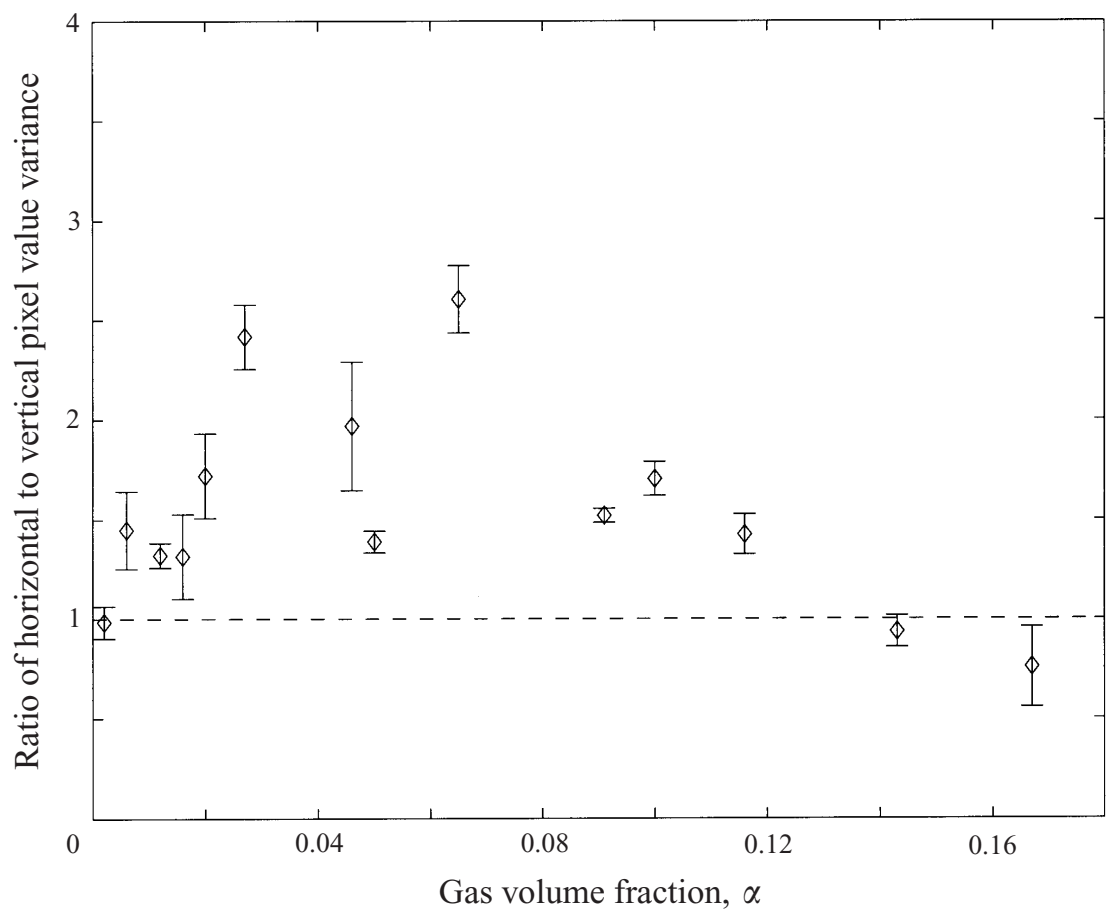

FIGURE 21. Ratio of the variances of the horizontally and vertically averaged pixel values $\Psi_{h} / \Psi_{v}$, as a function of gas volume fraction. The bars indicate the statistical error in the measurement. Each data point results from the processing of six photograph sections.

experiment is of the order of the channel gap width; hence, to better quantify the existence of clusters averages were performed over $1 \times 1 \mathrm{~cm}^{2}$ regions of the images. Figure 21 shows the ratio $\Psi_{h} / \Psi_{v}$ as a function of the gas volume fraction. Clearly, the figure shows that $\Psi_{h}$ is larger than $\Psi_{v}$ and the difference is largest for gas volume fractions in between 0.04 and 0.08 . The preferential structure found in the horizontal direction indicates that clustering is, in fact, occurring. The maximum of $\Psi_{h} / \Psi_{v}$ around $\alpha=0.05$ could result from the fact that the method of analysis is most capable of detecting bubble clustering at this intermediate volume fraction for which the image is neither nearly all white nor all black.

Note that strong clustering is expected to occur according to the criteria proposed by Spelt \& Sangani (1998) for the values of $T_{b} / u_{b}^{2}$ measured in this experiment. Although bubble clustering is indeed observed in the experiment, it is not to the extent observed by Sangani \& Didwania (1993) and Smereka (1993). In these studies the suspension formed horizontal rafts in which bubbles accumulated. Bands of bubbles and clear liquid were observed alternately extending through the entire computational domain. Only qualitative comparisons are possible; however, it is clear that the amount of clustering found in the experiment is not as strong as that observed in the simulations. In the simulations of Sangani \& Didwania and Smereka the bubble velocity variance was only that produced by non-deformable bubbles with potential flow interactions. The bubble velocity variance measured in our experiments was considerably larger and presumably arose due to bubble-wall interactions, deformability of the bubbles, and/or deviations from potential flow. Spelt \& Sangani provide a criterion for stability of suspensions with larger velocity variance but they do not quantify the amount of 
clustering that occurs in unstable suspensions. The more modest clustering seen in our experiments may result from the higher bubble velocity variance, the ability of the walls to enhance cluster breakup, or the non-sphericity of the bubbles. Duineveld (1994) observed that oblate bubbles with sufficiently large size and aspect ratio may tend to repel one another in the horizontal direction and this mechanism would inhibit cluster formation.

\section{Summary and final remarks}

In this paper, we have presented the first experimental results concerning the averaged behaviour of bubble suspensions that satisfy the conditions of large Reynolds number and moderately small Weber number necessary for comparison with theories based on potential flow interactions among the bubbles. Nearly monodisperse bubble suspensions were generated by a bank of identical capillaries. The flow properties were measured using two different probes and digital image processing. A hot-wire probe was used to measure the liquid velocity variance and the bubble-probe collision rate. The mean and variance of the bubble velocity and bubble-probe collision rate were measured using a dual impedance probe.

The mean bubble velocity was observed to decrease as the concentration increased, at a rate comparable to that predicted by Spelt \& Sangani (1998). The mean bubble velocity was found to decrease rapidly for very dilute suspensions, resulting from the bubble-wall interactions. The bubble velocity variance increased rapidly with gas volume fraction in very dilute suspensions and more moderately at higher concentrations.

A salient feature of the experiments presented in this paper is the effect of the containing walls. The ratio of the channel width to the bubble diameter was moderately large (about 13), so that one might have expected the bulk of the suspension to be relatively unaffected by the walls. However, the walls induce an oscillatory trajectory on single bubbles moving in the channel in which the bubbles translate to a position within a bubble diameter of one wall and then reverse direction to approach the other wall.

The bubble velocity variance measured in our experiments was too small to produce a stable suspension according to the criteria proposed by Spelt \& Sangani (1998), so we should expect to observe evidence of bubble clustering. Three types of experimental evidence for bubble clustering were presented. First, the fluid velocity variance was found to be about 10 times larger than that which would be produced by homogeneously distributed bubbles. Second, the power spectrum of the fluid velocity indicated that much of the energy of the fluid motion was at a frequency characteristic of the passage of bubble clusters with a size comparable with the channel diameter. Finally, direct visual evidence was obtained from video images such as that in figure 2 and the results of the image processing. The existence of horizontal clusters supports the theories for bubbles interacting through potential flow disturbances. The extent of the clustering observed in the experiments is considerably less than that seen in the simulations.

Although we observed mild bubble clustering in the present experiments, no largescale instabilities or macroscopic flows were observed. It has been noted by previous researchers (Lammers \& Biesheuvel 1996, for example) that large vortical structures appear in bubbly columns at sufficiently high gas volume fractions. These stronger inhomogeneities are thought to result from the instability of the suspension to void fraction waves with vertical wave vectors. The postulated instability mechanism involves the dependence of the bubble drag on volume fraction and the inertia 
associated with the bubbles' added mass. Vortical structures moving up and down and extending across the channel width were observed in our apparatus for concentrations larger than 0.20. Zenit et al. (2000) observed that such instabilities occur at lower concentrations for the case of gravity-driven shear flows in inclined channels. The criterion for the onset of such instabilities in vertical and inclined channels filled with high-Reynolds-number, low-Weber-number bubbles is an interesting topic for future research.

This work was supported by NASA under grant number NAG3-1853. The initial work on the design of the flow cell and the method of detecting bubble collisions with hot film probes was performed by Reghan J. Hill and Thulasidas Chelppannair, respectively.

\section{REFERENCES}

Biesheuvel, A. \& Gorissen, W. C. M. 1990 Void fraction disturbances in a uniform bubbly fluid. Intl J. Multiphase Flow 16, 211-231.

Biesheuvel, A. \& WiJngaARden L. van 1982 The motion of pairs of gas bubble in a perfect liquid. J. Engng Maths 16, 349-365.

Bruun, H. H. 1995 Hot-Wire Anemometry, Principles and Signal Analysis. Oxford.

Bulthuis, H. F., Prosperetti, A. \& Sangani, A. S. 1995 Particle stress in disperse two-phase potential flow. J. Fluid Mech. 294, 1-16.

Ceccio, S. L. \& Georges, D. L. 1996 A review of electrical impedance techniques for the measuremet of multiphase flows. Trans. ASME: J. Fluids Engng 118, 391-399.

Clift, R., Grace, J. R. \& Weber, M. E. 1978 Bubbles, Drops, and Particles. Academic.

Duineveld, P. C. 1994 Bouncing and coalescence of two bubbles in water. PhD thesis, Universiteit Twente.

Duineveld, P. C. 1995 The rise and shape of bubbles in pure water at high Reynolds number. J. Fluid Mech. 292, 325-332.

Durlofsky, L. \& Brady, J. F. 1988 The sedimentation rate of disordered suspensions. Phys. Fluids 31, 717-727.

ISHII, M. \& ZUBER, N. 1979 Drag and relative velocity in bubbly, droplet or particulate flows. AIChE J. 25, 843-855.

Kang, S. A., Sangani, A. S., Tsao, H.-K. \& Koch, D. L. 1997 Rhelogy of dense bubble suspensions. Phys. Fluids 9, 1540-1570.

KoK, J. B. W. 1993 Dynamic of a pair of gas bubbles moving through a liquid; Part I: Theory. Eur. J. Mech. B/Fluids 251, 541-560.

Kumaran, V. \& KoCH, D. L. 1994 The effect of hysrodynamic interactions on the averaged properties of a bidisperse suspension of high Reynolds number, low Weber number bubbles. Phys. Fluids A 5, 1135-1140.

LADD, A. J. C. 1990 Hydrodynamic transport coefficients of random dispersions of hard spheres. J. Chem. Phys 93, 3484-3494.

Lammers, J. H. \& Biesheuvel, A. 1996 Concentration waves and the instability of bubbly flows. J. Fluid Mech. 328, 67-93.

LESSARD, R. R. \& ZiEMINSKI, S. A. 1971 Bubble coalescence and gas transfer in electrolitic aqueous solutions. Ind. Engng Chem. Fundam. 10, 260-269.

Lin, T.-J., ReEse, J., Hong, T. \& FAN, L.-S. 1996 Quantitative analysis and computations of two dimensional bubble comuns. AIChE J. 42, 301-318.

LiU, T. J. \& BAnkofF, S. G. 1993 Structure of air-water bubbly flow in a vertical pipe-II. Void fraction, bubble velocity and bubble size distribution. Intl J. Heat Mass Transfer 36, 1061-1072.

Molerus, O. \& KurTin, M. 1985 Hydrodynamics of bubble columns in the uniform bubbling regime. Chem. Engng Sci. 40, 647-652.

Moore, D. W. 1963 The boundary layer on a spherical gas bubble. J. Fluid Mech. 16, 161-176.

Moore, D. W. 1965 The velocity of rise of distorted gas bubbles in a liquid of small viscosity. J. Fluid Mech. 23, 749-749. 
OG̃uz, H. N. \& Prosperetti, A. 1993 Dynamics of bubble growth and detachment from a needle. J. Fluid Mech. 257, 111-145.

Richardson, J. F. \& ZAKI, W. N. 1954 Sedimentation and fluidisation: Part I. Trans. Inst. Chem. Engrs 32, 35-53.

Sangani, A. S. \& Didwania, A. K. $1993 a$ Disperse phase stress tensor in flows of bubbly liquids at large Reynolds numbers. J. Fluid Mech. 248, 27-54.

Sangani, A. S. \& Didwania, A. K. $1993 b$ Dynamics simulations of flows of bubbly liquids at large Reynolds numbers. J. Fluid Mech. 250, 307-337.

Smereka, P. 1993 On the motion of bubbles in a periodic box. J. Fluid Mech. 254, 79-112.

Spelt, P. D. M. \& SANGani, A. S. 1998 Properties and averaged equations for flows of bubbly liquids. Appl. Sci. Res. 58, 337-386.

Tsao, H.-K. \& Koch, D. L. 1994 Collisions of slightly deformable, high Reynolds number bubbles with short range repulsive forces. Phys. Fluids 6, 671-690.

TsaO, H.-K. \& KoCH, D. L. 1997 Observations of high Reynolds number bubbles interacting wityh a rigid wall. Phys. Fluids 9, 2591-2600.

Valukina, N. V., Koz'menko, B. K. \& Kashinski 1979 Characteristics of a flow of monodisperse gas-liquid mixture in a vertical tube. Inzhenerno-Fizicheskii Zhurnal 36, 695-698.

Waniewski, T. A. 1999 Air entrainment by plunging bow waves. PhD Thesis, California Institute of Technology.

WijngaArden, L. van 1998 On pseudo turbulence. Theor. Comput. Fluid Dyn. 10, 449-458.

WiJngaARDEN, L. van \& KAPTEYN, C. 1990 Concentration waves in dilute bubble/liquid mixtures. J. Fluid Mech. 212, 111-137.

Yurkovetsky, Y. \& Brady, J. F. 1994 Statistical mechanics of bubbly liquids. Phys. Fluids 8, $881-895$.

Zenit, R., Koch, D. L. \& SAngani, A. S. 2000 Shear flow of a suspension of bubbles rising in an inclined channel. Under consideration for publication in $J$. Fluid Mech.

Zhang, D. Z. \& Prosperetti, A. 1994 Averaged equations for inviscid disperse two-phase flow. J. Fluid Mech. 267, 185-219. 